\title{
Acylsugars Protect Nicotiana Benthamiana Against Insect Herbivory and Desiccation
}

\section{Honglin Feng}

Boyce Thompson Institute for Plant Research

\section{Lucia Acosta-Gamboa}

Cornell University

\section{Lars H Kruse}

Cornell University

Jake D Tracy

Cornell University

\section{Seung Ho Chung}

Boyce Thompson Institute for Plant Research

\section{Alba Ruth Nava Fereira}

The University of Texas at San Antonio

\section{Sara Shakir}

Boyce Thompson Institute for Plant Research Hongxing Xu

Boyce Thompson Institute for Plant Research

\section{Garry Sunter}

The University of Texas at San Antonio

\section{Michael A Gore}

Cornell University

\section{Clare L Casteel}

Cornell University

\section{Gaurav D. Moghe}

Cornell University

Georg Jander ( $\nabla$ gj32@cornell.edu )

Boyce Thompson Institute For Plant Research https://orcid.org/0000-0002-9675-934X

\section{Research Article}

Keywords: acylsugar, aphid, ASAT, desiccation, Nicotiana benthamiana, whitefly 
DOI: https://doi.org/10.21203/rs.3.rs-596878/v1

License: (c) (1) This work is licensed under a Creative Commons Attribution 4.0 International License. Read Full License 
Article Title

Acylsugars protect Nicotiana benthamiana against insect herbivory and desiccation

Author names

Honglin Feng ${ }^{\mathrm{a}}$, Lucia Acosta-Gamboa ${ }^{\mathrm{b}}$, Lars H. Kruse ${ }^{\mathrm{c}, \mathrm{f}}$, Jake D. Tracy ${ }^{\mathrm{d}, \mathrm{g}}$, Seung Ho Chung ${ }^{\mathrm{a}}$, Alba Ruth Nava Fereira ${ }^{\mathrm{e}}$, Sara Shakir ${ }^{\mathrm{a}, \mathrm{h}}$, Hongxing Xu ${ }^{\mathrm{a}, \mathrm{i}}$, Garry Sunter ${ }^{\mathrm{e}}$, Michael A. Gore ${ }^{\mathrm{b}}$, Clare L. Casteel ${ }^{\mathrm{d}}$, Gaurav D. Moghe ${ }^{\mathrm{c}}$, Georg Jander ${ }^{\mathrm{a}^{*}}$

Author Affiliations

12 aBoyce Thompson Institute, Ithaca NY, USA

13 bPlant Breeding and Genetics Section, School of Integrative Plant Science, Cornell University, Ithaca NY, 14 14853, USA

15 'Plant Biology Section, School of Integrative Plant Science, Cornell University, Ithaca NY, 14853, USA

16 dPlant-Microbe Biology and Plant Pathology Section, School of Integrative Plant Science, Cornell

17 University, Ithaca NY, 14853, USA

18 'Department of Biology, University of Texas San Antonio, San Antonio TX, 78249, USA

19 fPresent address: Michael Smith Laboratories, University of British Columbia, Vancouver, BC, V6T 1Z4,

20 Canada

21 'Present address: Department of Plant Sciences \& Plant Pathology, Montana State University, Bozeman

22 MT, 59717, USA

23 hPresent address: Gembloux Agro-Bio Tech Institute, the University of Liege, Gembloux, Belgium

24 iPresent address: College of Life Science, the Shaanxi Normal University, Xi'an, China

\section{*Correspondence:}

Georg Jander

Boyce Thompson Institute

Ithaca, NY 14853

USA

Phone: 607-254-1365

Email: gj32@cornell.edu

ORCID: 0000-0002-9675-934X 
Key Message:

37 Nicotiana benthamiana acylsugar acyltransferase (ASAT) is required for protection against desiccation

38 and insect herbivory, and knockout mutations provide a new resource for investigation of plant-aphid and

39 plant-whitefly interactions.

Abstract

42 Nicotiana benthamiana is used extensively as a transient expression platform for functional analysis of

43 genes from other species. Acylsugars, which are produced in the trichomes, are a hypothesized cause of

44 the relatively high insect resistance that is observed in $N$. benthamiana. We characterized the $N$.

45 benthamiana acylsugar profile, bioinformatically identified two acylsugar acyltransferase genes, ASAT1

46 and ASAT2, and used CRISPR/Cas9 mutagenesis to produce acylsugar-deficient plants for investigation

47 of insect resistance and foliar water loss. Whereas asat 1 mutations reduced accumulation, asat 2 mutations

48 caused almost complete depletion of foliar acylsucroses. Three hemipteran and three lepidopteran

49 herbivores survived, gained weight, and/or reproduced significantly better on asat2 mutants than on

50 wildtype $N$. benthamiana. Both asat 1 and asat 2 mutations reduced the water content and increased leaf

51 temperature. Our results demonstrate the specific function of two ASAT proteins in N. benthamiana

52 acylsugar biosynthesis, insect resistance, and desiccation tolerance. The improved growth of aphids and

53 whiteflies on asat 2 mutants will facilitate the use of $N$. benthamiana as a transient expression platform for

54 the functional analysis of insect effectors and resistance genes from other plant species. Similarly, the

55 absence of acylsugars in asat 2 mutants will enable analysis of acylsugar biosynthesis genes from other

56 Solanaceae by transient expression.

57

58 Keywords: acylsugar, aphid, ASAT, desiccation, Nicotiana benthamiana, whitefly 


\section{Introduction}

60 Nicotiana benthamiana, a wild tobacco species that is native to Australia, is commonly employed by

61 plant molecular biologists as platform for investigating plant-virus interactions (Goodin et al., 2008),

62 expression of transgenes using viral and bacterial vectors (Bally et al., 2018), and overproducing proteins

63 and small molecules for subsequent purification (Arntzen, 2015; Powell, 2015). Although $N$.

64 benthamiana has been used extensively for studying plant-virus and plant-bacterial interactions, is not a

65 very suitable host for tobacco feeding generalist herbivores, in particular Hemiptera such as Myzus

66 persicae (green peach aphid; Thurston, 1961; Hagimori et al., 1993) and Bemisia tabaci (whitefly; Simon

67 et al., 2003).

68 The poor growth of generalist insect herbivores on $N$. benthamiana may be attributed in part to

69 glandular trichomes. These epidermal secretory structures on the leaf surface of $\sim 30 \%$ of vascular plants

70 (Weinhold and Baldwin, 2011; Glas et al., 2012) have been found to play a crucial defensive role in

71 several ways: as a physical obstacle for insect movement (Cardoso, 2008), entrapment (Simmons et al.,

72 2004), synthesis of volatiles and defensive metabolites (Laue et al., 2000; Schilmiller et al., 2010; Glas et

73 al., 2012), and production of herbivore-resistant proteins (e.g. T-phylloplanin; Shepherd and Wagner,

74 2007). In addition, glandular trichomes also protect plants from abiotic stresses like transpiration water

75 loss and UV irradiation (Karabourniotis et al., 1995).

76 There are two main types of glandular trichomes on $N$. benthamiana leaves, large swollen-stalk

77 trichomes and small trichomes that are capped by a secretory head with one, two, or four cells. The large

78 trichomes have been shown to secrete phylloplane proteins in N. tabacum. The small trichomes, which are

79 the most abundant on tobacco leaf surfaces, secrete exudates, including acylsugars (Wagner et al., 2004;

80 Slocombe et al., 2008).

81 Acylsugars, generally sucrose or glucose esterified with aliphatic acids of different chain lengths

82 (Fig. 1), are abundant insect-deterrent metabolites (Arrendale et al., 1990; Slocombe et al., 2008; Moghe

83 et al., 2017). Specific acylsugars, which were associated with aphid-resistant Nicotiana species, were not

84 detected in susceptible species in this genus (Hagimori et al., 1993). Relative to cultivated tomatoes

85 (Solanum lycopersicum), acylsugars found in wild tomatoes (Solanum pennellii) were associated with

86 greater resistance against M. persicae and B. tabaci (Rodriguez et al., 1993; Marchant et al., 2020). The

87 synthetic sucrose octanoate (an analog of Nicotiana gossei sugar esters) was effective in the field against

88 Asian citrus psyllids (Diaphorina citri), citrus leafminer (Phyllocnistis citrella), and a mite complex

89 (including Texas citrus mite, red spider mite, and rust mite) (McKenzie and Puterka, 2004; McKenzie et

90 al., 2005).

91 Acylsugars and leaf surface lipids more generally may contribute to plant drought tolerance.

92 Transcriptomic studies of drought-tolerant S. pennellii populations showed that lipid metabolism genes 
93 were among those that are most responsive to drought stress (Gong et al., 2010; Egea et al., 2018), and

94 high acylsugar abundance was associated with drought tolerance (Fobes et al., 1985). Similarly, acylsugar

95 abundance was correlated with drought tolerance in Solanum chilense (O' Connell et al., 2007) and

96 Nicotiana obtusifolia (Kroumova et al., 2016). Although the mechanism is not completely understood, it

97 has been proposed that the polar lipids reduce the surface tension of adsorbed dew water, thereby

98 allowing the leaves absorb more condensed water (Fobes et al., 1985).

99 Recently, enzymes involved in acylsugar biosynthesis have been identified. Four acylsugar

100 acyltransferases (ASATs), SlASAT1, SlASAT2, SlASAT3, and SlASAT4, have been biochemically

101 characterized in cultivated tomato (Fan et al., 2016). SlASAT1 catalyzes the first step of sucrose

102 acylation, using sucrose and acyl-CoA to generate monoacylsucroses via pyranose $\mathrm{R}_{4}$ acylation. SlASAT2

103 uses the product of SlASAT1 and acyl-CoA to generate diacylsucroses. Further, SlASAT3 uses the

104 diacylsucroses generated by SIASAT2 to make triacylsucroses by acylating the diacylsucrose five-

105 membered (furanose) ring (Fan et al., 2016). Then, SlASAT4 (formerly SlASAT2), specifically expressed

106 in the trichomes, makes tetraacylsucroses by acetylating triacylsucroes using C2-CoA (Schilmiller et al.,

107 2012). The expression, activity, and even the order of ASATs in the biosynthetic pathway varies among

108 different plant species, which likely contributes to the observed trichome chemical diversity (Kim et al., 109 2012).

110 ASATs have been studied most intensively in tomato, and are annotated in other available

111 Nicotiana genomes (Gaquerel et al., 2013; Van et al., 2017; Egan et al., 2019). However, ASAT genes in

$112 N$. benthamiana were not previously annotated or functionally characterized. Therefore, the goal of

113 current study was to investigate the role of acylsugars in protecting $N$. benthamiana against insect feeding

114 and foliar water loss, as well as to create an insect-susceptible ASAT mutant line to facilitate use of $N$.

115 benthamiana for research on plant-insect interactions. We bioinformatically identified two ASAT genes in

$116 N$. benthamiana, NbASAT1 and NbASAT2. Using CRISPR/Cas9 to create NbASAT1 and NbASAT2 mutant

117 lines, we showed that knockout mutations reduced acylsugar content, decreased resistance to six

118 generalist insect herbivores [M. persicae, B. tabaci, Macrosiphum euphorbiae (potato aphid), Helicoverpa

$119 z e a$ (corn earworm), Heliothis virescens (tobacco budworm), and Trichoplusia ni (cabbage looper)], and

120 increased foliar water loss

121

122 Results

123 Identification of ASAT1 and ASAT2 in N. benthamiana

124 Using reciprocal comparisons to confirmed Solanaceae ASAT genes (Moghe et al., 2017), we identified

125 three highly homologous sequences in the $N$. benthamiana genome: Niben101Scf02239Ctg025,

126 Niben101Scf22800Ctg001, and Niben101Scf14179Ctg028 (gene identifiers are from annotations at 
127 solgenomics.net) (Bombarely et al., 2012). Whereas Niben101Scf02239Ctg025 and

128 Niben101Scf22800Ctg001 were annotated as a full-length coding sequences with strong coverage in

129 available RNAseq datasets, Niben101Scf14179Ctg028 was annotated as a pseudogene because it appears

130 to be a fragment of the predicted cDNA Niben101Scf141790g02010.1 with no coverage in available

131 RNAseq datasets. In a more recently assembled N. benthamiana genome (Schiavinato et al., 2019), the

132 Niben101Scf02239Ctg025 and Niben101Scf22800Ctg001 sequences were confirmed, the pseudogene

133 Niben101Scf14179Ctg028 was annotated as part of Niben101Scf02239Ctg025, and there were no

134 additional annotated ASAT candidates.

135 To infer ASAT evolution and function, we constructed a protein phylogenetic tree of previously

136 annotated Solanaceae ASATs (Figs. 2, S1, S2; Tables S1, S2). In this tree, Niben101Scf02239Ctg025

137 formed a monophyletic group with other ASATs including the biochemically characterized SsASAT1,

$138 P a A S A T 1$ and HnASAT1. Therefore, we named Niben101Scf02239Ctg025 as N. benthamiana ASAT1

139 (NbASAT1). Niben101Scf22800Ctg001 formed a monophyletic group with other ASATs including the

140 biochemically characterized NaASAT2, HnASAT2, and PaASAT2. Therefore, we named

141 Niben101Scf22800Ctg001 as $N$. benthamiana ASAT2 (NbASAT2). Notably, the ASAT2 monophyletic

142 group also included the biochemically characterized SpASAT1, SlASAT1, and SnASAT1 (Fig. 2).

\section{Generation of ASAT mutants}

145 Using CRISPR/Cas9 coupled with tissue culture, we obtained two independent homozygous mutants for 146 both NbASAT1 and NbASAT2. asat1-1 has a five-nucleotide deletion at the gRNA3 cutting site and a 147 single-nucleotide insertion at the gRNA2 cutting site, leading to a frameshift between gRNA3 and 148 gRNA2. asat1-2 has a 318-nucleotide deletion between the gRNA3 and gRNA2 cutting sites (Fig. 3a).

149 asat2- 1 has a single-nucleotide deletion at the gRNA3 cutting site and single-nucleotide insertion at the 150 gRNA2 cutting site, leading to a frameshift between the two sites. asat2-2 has a 115-nucleotide deletion 151 at the gRNA3 cutting site and a single-nucleotide insertion at the gRNA2 cutting site (Fig. 3b).

152 Even though ASAT1 and ASAT2 are located on different scaffolds in the $N$. benthamiana genome 153 assembly (Schiavinato et al., 2019), we were not able to find homozygous asat1 asat2 double mutants 154 among $40 \mathrm{~F} 2$ progeny from crosses between asat1 and asat2 plants. Similarly, when we transformed $N$.

155 benthamiana in tissue culture with gRNA targeting both ASAT1 and ASAT2, simultaneously or 156 sequentially, we identified each individual knockout mutation, but no homozygous double mutants.

157 Although we cannot completely rule out other scenarios, it is possible that asat1 asat2 double mutations 158 are deleterious or lethal for $N$. benthamiana. 
In the LC/MS profile of $N$. benthamiana leaf surface washes, we characterized twelve mass features as acylsucroses based on their characteristic peaks and neutral losses. Those twelve $m / z$ ratios included 383.12, 467. 21, 509.22, 555.23, 593.32, 621.31, 625.31, 635.32, 639.32, 667.32, 671.30, 681.34 (Fig. S3a). In negative electron spray ionization mode, the characteristic peak features included $\mathrm{m} / \mathrm{z}$ of 341.11 for sucrose, 509.22 for sucrose $+\mathrm{C} 2+\mathrm{C} 8,467.21$ for sucrose $+\mathrm{C} 8,495.21$ for sucrose $+\mathrm{C} 7$, and 383.12 for sucrose $+\mathrm{C} 2$; the neutral loss peaks included mass for 126.10 for C8 (acyl-chain with 8 carbons), 129.09 for $\mathrm{C} 7+\mathrm{H}_{2} \mathrm{O}$, and 59.01 for $\mathrm{C} 2+\mathrm{H}_{2} \mathrm{O}$ (Fig. S3b). Based on their MS/MS peak features, retention times and relative abundances, we predicted that the identified mass features are mainly derived from two acylsucroses as formate or chloride adducts, pathway intermediates, and/or resulted from in-source fragmentation. We named the two acylsucroses S3:17(2,7,8) and S3:18(2,8,8) (in the nomenclature, "S" refers to the sucrose backbone, "3:18" indicates three acyl chains with total eighteen carbons, and the length of each acyl chain is shown in parentheses) (Fig. 1; S3a).

In wildtype plants, $\mathrm{S} 3: 18(2,8,8)$ is the dominant acylsucrose, whereas $\mathrm{S} 3: 17(2,7,8)$ has relatively

174 low abundance (Fig. 4). S2:16(8,8) and S2:15(7,8), which may be biosynthetic pathway intermediates for

$175 \mathrm{~S} 3: 18(2,8,8)$ and S3:17(2,7,8), respectively, are present at lower levels. Compared to wildtype $N$. benthamiana, both asat2-1 and asat2-2 were almost completely depleted in both S3:17(2,7,8) and $\mathrm{S} 3: 18(2,8,8)$, as well as in the two predicted biosynthetic intermediates $\mathrm{S} 2: 15(7,8)$ and $\mathrm{S} 2: 16(8,8)$. For asat $1-1$ and asat 1-2, the detected acylsucroses were less abundant and significantly reduced only in asat1-1 (Fig. 4). Although acylsugar content was reduced in the ASAT mutants, the structure and abundance of trichomes on the leaf surface were not visibly changed (Fig. S4).

\section{Insect performance is improved on ASAT2 mutant lines}

183 To test the role of acylsugars in protecting $N$. benthamiana against insect pests, we started with

184 synchronized first-instar M. persicae to monitor aphid survival and growth over time. Significant

185 improvements in aphid survivorship were observed as early as at 2 days post-feeding $(p<0.001)$ on the

186 asat2-1 and asat2-2 mutants and increased until the end of the 5-day monitoring period $(p<0.001)$ (Fig.

187 5a; Table S3). After 5 days of feeding, surviving aphids on both asat1 and asat2 plants were larger than

188 those on wildtype plants (Fig. 5b). When we measured progeny production by five adult aphids over a

189 period of seven days, an average of more than 200 nymphs were produced on the asat2 mutants,

190 significantly more than the number of nymphs produced on either wildtype or asat1 mutants $(p<0.05$,

191 Fig. 5c). In aphid choice assays, a preference for asat2-1 and asat2-2 leaves was consistently observed in 192 any pairwise combination with wildtype, asat1-1, and asat1-2 leaves ( $p<0.001$, Chi-square test, Fig.

193 5d,e; S5). No M. persicae preference was observed between wildtype $N$. benthamiana and asat 1 mutants $194 \quad(p>0.05$, Fig. 5f; S5). 
When aphid colonies were allowed to grow long-term on asat2-1 mutant and wildtype $N$. benthamiana in the same cage, there were many more aphids on the mutant plants (Fig. S6a,b), likely

197 resulting from a combination of host plant choice and increase growth on the asat2-1 mutant. It is

198 noteworthy that, on the asat2-1 mutant plants, aphids were feeding on the more nutritious younger leaves, 199 which tend to be better-defended in plants. By contrast, on wildtype $N$. benthamiana aphids were only 200 were able to feed on older, senescing leaves and were primarily on the abaxial surface. Consistent with 201 the increased aphid presence, growth of the asat2-1 mutant plants were visibly reduced relative to 202 wildtype $N$. benthamiana (Fig. S6b). Given the almost identical phenotypes of asat2-1 and asat2-2 203 mutants, subsequent insect assays were conducted with T2 progeny of the asat2-1 line.

As we observed with $M$. persicae, the asat2-1 mutation improved M. euphorbiae performance on $N$. benthamiana (Fig. 6a-c). Significantly increased M. euphorbiae survival was observed after $24 \mathrm{~h}$ on asat $2-1$ compared to wildtype $(p<0.001$, Fig. 6a). Additionally, significantly more nymphs were produced by adult $M$. euphorbiae in the course of $24 \mathrm{~h}$ on asat $2-1$ than on wildtype ( $p<0.05$, Fig. 6 b). In choice assays, potato aphids preferentially chose asat2-1 leaves over wildtype leaves $(p<0.001$, Fig. 6c). Whereas we were not able to establish an M. euphorbiae colony on wildtype $N$. benthamiana, the aphids readily formed colonies on the asat2-1 mutant plants (Fig. 6d).

Survival of B. tabaci adults was greatly increased on the asat2-1 mutant relative to wildtype $N$.

212 benthamiana (Fig. 6e). Moreover, whiteflies laid significantly fewer eggs on wildtype than on asat2-1

213 mutant plants over three days (Fig. 6f). Dead adult whiteflies were observed on wildtype plants (Fig.

214 S7a), and it was not possible to establish a reproducing colony. By contrast, after 23 days of feeding,

215 whiteflies of different life stages were observed on asat2-1 mutant plants (Fig. S7b-d). In choice assays

216 with mutant and wildtype plants in the same cage, whiteflies preferentially settled on asat2-1 plants

217 (72\%) over wildtype (26\%) in a 24-h experiment (Fig. 6g). Notably, after $24 \mathrm{~h}$, all whiteflies on asat2-1

218 were alive, whereas about half of the whiteflies that had settled on the wildtype plants were dead (Fig. 6f).

219 To determine whether depletion of acylsugars in ASAT2 mutants improves the performance of

220 generalist lepidopteran herbivores on $N$. benthamiana, we conducted experiments with $H$. zea, $H$.

221 virescens, and T. ni. When neonates were placed on the leaves of wildtype or asat2-1 mutant, no H. zea

222 caterpillars were recovered (Fig. 7a). Survivorship of $H$. virescens and $T$. ni larvae on $N$. benthamiana

223 was low, and the mass of the surviving larvae after ten days was not significantly increased on the mutant

224 relative to wildtype (Fig. 7b,c). Due to the low survival of neonates, we repeated the caterpillar bioassay

225 using five-day-old larvae that had been reared on artificial diet. Almost all H. zea and H. virescens larvae

226 survived for seven days on wildtype and asat2-1 mutant plants, and survival of T. ni caterpillars was

227 higher on asat2-1 than on wildtype plants (Fig. 7d-f). The relative growth rates of surviving $H$. zea, $H$. 
virescens, and $T$. $n i$ larvae were higher on the asat $2-1$ mutant by $35 \%, 47 \%$, and $99 \%$, respectively, than on wildtype plants (Fig. 7d-f).

\section{Water loss is greater in asat 2 mutant plants than in wildtype}

232 While conducting aphid choice assays with detached leaves (Fig. 5d-f; 6c), we noticed that the mutant 233 leaves dried out faster than wildtype leaves. This effect was quantified using detached-leaf assays, in 234 which asat2 leaves lost significantly more water over $24 \mathrm{~h}$ than leaves from either wildtype or asat 1 235 mutant (Fig. 8a; S8a). Using hyperspectral imaging, we determined that the leaf water content of intact 236 plants, as measured by the water band index (WBI), was significantly lower in asat2 mutants than in 237 wildtype (Fig. 8b; S8b). Although the asat1 mutants did not lose water faster than wildtype in detached 238 leaf assays (Fig. 8a), the leaf water content in asat1 mutants was significantly lower than wildtype (Fig.

$239 \mathbf{8 b} ; \mathbf{S 8 b})$. Measurement of leaf temperature by thermal imaging showed that, consistent with the reduced 240 leaf water content, the leaf temperature of the acylsugar mutants was significantly higher than that of 241 wildtype plants (Fig. 8c; S8c).

\section{Discussion}

244 We identified only two ASAT genes, NbASAT1 and NbASAT2, and a fragmented pseudogene, in the $N$. benthamiana genome (Table S2). By contrast, in other Nicotiana species, there are more predicted ASATs, e.g. one ASAT1, one ASAT2, and 20 ASAT3-like genes in N. attenuata (Gaquerel et al., 2013; Van et al., 2017), 35 ASAT3-like genes in N. tabacum, and 19 ASAT3-like genes in N. tomentosiformis (Egan et al., 2019). Given the small number of predicted ASAT genes in N. benthamiana, other enzymes may also be involved in acylsugar biosynthesis. For instance, knockdown of E1- $\beta$ branched-chain $\alpha$-keto acid dehydrogenase significantly reduces acylsugars in N. benthamiana (Slocombe et al., 2008). Additionally, Isopropylmalate Synthase 3 in cultivated and wild tomatoes (Ning et al., 2015) and Acyl-Sucrose FructoFuranosidase 1 in wild tomato (Leong et al., 2019) encode enzymes that are involved in determining acylsugar composition. Further studies will be needed to characterize other genes involved in $N$.

254 benthamiana acylsugar biosynthesis.

Acylsugars can be categorized as sucrose or glucose esters based on the sugar cores, which are decorated with varying numbers or lengths of acyl chains (Kim et al., 2012). Whereas some wild tomatoes produce a mixture of acylsucroses and acylglucoses, we observed only acylsucroses (Fig. 1; 4), 258 consistent with previous identification of these compounds in N. benthamiana (Matsuzaki et al., 1989; 259 Matsuzaki et al., 1992; Hagimori et al., 1993; Slocombe et al., 2008). Nevertheless, it has been reported 260 that $N$. benthamiana produces acylglucoses, although in lower abundance than acylsucroses (Hagimori et 261 al., 1993), and one acylglucose structure has been proposed (Matsuzaki et al., 1992). Our failure to detect 
262 acylglucoses may be explained by the use of different isolates of $N$. benthamiana, growth conditions, 263 plant stage, and/or the detection methods. Whereas we used 1-month-old plants and LC/MS, Matsuzaki 264 et al. (1992) used 3-month-old plants and GC/MS to detect acylglucoses in N. benthamiana.

265 The abundance of the characterized acylsugars was reduced to a greater extent in N. benthamiana 266 asat2 than in asat1 mutants (Fig. 4). This suggests that either NbASAT2 functions upstream of NbASAT1 267 in the acylsugar biosynthesis pathway, but partially complements NbASAT1 activity, or NbASAT1 and 268 NbASAT2 have similar functions in the biochemical pathway, but NbASAT2 had higher abundance or 269 enzymatic activity. It is not known whether $N$. benthamiana ASATs are monomeric or multimeric, but 270 BAHD acyltransferases generally are monomeric enzymes (D'Auria, 2006), suggesting that heterodimers 271 between ASAT1 and ASAT2 are unlikely to affect the observed phenotypes. In the ASAT phylogenetic tree (Fig. 2), NbASAT2 is closely related to some biochemically characterized ASAT1 proteins in other Solanaceae species, including the SpASAT1, SlASAT1, and SnASAT1. Those ASAT1s have some substrate overlap with the ASAT2s found in the corresponding species, indicating that ASAT2 has moved toward utilizing the ASAT1 substrate in these species over time (Moghe et al., 2017). The final activity shift that has become fixed in the Solanum genus, most likely occurred after the divergence of the Solanum and Capsicum clades (Moghe et al., 2017). However, if our hypothesis of partial complementation of $N b$ ASAT1 by $N b$ ASAT2 is correct, it may flag a transition stage or suggest independent Nicotiana-specific evolution of the ASAT1 and ASAT2 functions. Based on previous knowledge of BAHD activities (Moghe et al., 2017), we postulate that S2:15 (7,8) and S2:16 $(8,8)$ are produced by $N b A S A T 1$ and $N b A S A T 2$, whereas the acetylation is carried out by another unrelated BAHD enzyme - not unlike the distantly related SlASAT4 and Salpiglossis sinuata ASAT5 (Schilmiller et al., 2015; Moghe et al., 2017). Further characterization will be required to identify specific acyltransferase activities in $N$. benthamiana.

The observed role of acylsugars in protecting against desiccation (Fig. 8; S8) is consistent with reports from other Solanaceae (Fobes et al., 1985; O’ Connell et al., 2007; Kroumova et al., 2016), though this has not previously been verified with isogenic mutant and wildtype plants. In N. benthamiana, the drought-protective function of acylsugars is likely an adaptation to the seasonally arid native habitat in northwestern Australia (Goodin et al., 2008; The Australasian Virtual Herbarium, https://avh.ala.org.au). Relative to asat1 mutants, the lower acylsugar content of asat2 mutants (Fig. 4), resulted in more rapid water loss in detached leaves (Fig. 8a; S8a). However, despite the only partial decrease in the acylsugar content of asatl mutants, the decreases in water content and increases in leaf temperature of intact plants were similar to those of asat2 mutants (Fig. 8b,c; S8b,c).

Acylsugars with $\mathrm{C}_{7-12}$ chains have been shown to be the most toxic sugar esters for small phloemfeeding Hemiptera such as aphids, Asian citrus psyllids, and whiteflies (Chortyk et al., 1996; McKenzie 
and Puterka, 2004; Song et al., 2006). Synthetic acylsucroses with di-heptanoic acid (C7), di-octanoic acid (C8), and di-nonanoic acid (C9) acyl groups showed the highest mortality in bioassays with $M$. persicae and B. tabaci (Chortyk et al., 1996; McKenzie and Puterka, 2004; Song et al., 2006). Nicotiana gossei, a tobacco species that produces mainly C7-C8 acyl group acylsugars, has a high level of insect resistance relative to close relatives with acylsugar profiles that are not dominated by those with C7-C12 acyl groups (Thurston, 1961; Kroumova and Wagner, 2003). In N. benthamiana, the two most abundant acylsugars that we found contain $\mathrm{C} 7$ and predominantly $\mathrm{C} 8$ acyl groups, which is consistent with previous findings of mainly 5- and 6-methyl heptanoate (C8) in N. benthamiana (Kroumova and Wagner, 2003; Slocombe et al., 2008) and N. alata (Moghe et al., 2017). The almost complete depletion of acylsugars in our asat 2 mutants improved both hemipteran and lepidopteran performance, suggesting that the identified C8 acyl group acylsugars are providing insect resistance for $N$. benthamiana. performance on acylsugar-depleted $N$. benthamiana. Specialized metabolites in other plants, for instance glucosinolates in Arabidopsis thaliana (Clay et al., 2009) and benzoxazinoids in Zea mays (Meihls et al., 2013), regulate callose deposition as a secondary defense response. It is not known whether acylsugars contribute to the regulation of other defense responses in $N$. benthamiana. The observation of numerous dead whiteflies on wildtype $N$. benthamiana plants in choice assays (Fig. 6g), despite the option of moving to presumably more desirable asat2-1 mutant plants in the same cage, suggests that the acylsugars stickiness also plays a role in plant defense by immobilizing the insects. Both altered leaf turgor and leaf temperature (Fig. 8b,c) could affect insect feeding behavior and growth rate, though the specific effects on the six tested insect species cannot be determined without further research.

Although $H$. zea, $H$. virescens, and $T$. ni larvae grow well on cultivated tobacco, neonate larvae had a low survival rate on both wildtype and asat2-1 N. benthamiana (Fig. 7a-c). There was a higher survival rate with five-day-old larvae of the three tested species, which all grew significantly better on asat2-1 mutants than on wildtype $N$. benthamiana (Fig. 7d-f). Thus, $N$. benthamiana acylsugars likely provide at least some protection against lepidopteran pests. However, the high mortality of neonate larvae on asat2-1 plants suggests that either residual acylsugars or as yet unknown resistance mechanisms in $N$. benthamiana can provide protection. Additional mutations that decrease insect resistance, perhaps regulatory genes such as $\mathrm{COII}$ or genes affecting the production of other specialized metabolites, will be necessary to facilitate $N$. benthamiana experiments with $H$. zea, $H$. virescens, $T$. ni, and other commonly studied lepidopteran species.

The high mortality of hemipteran pests such as M. persicae and B. tabaci on wildtype $N$.

328 benthamiana (Fig. 5, 6) makes it challenging to interpret insect bioassays involving the transient 329 expression of heterologous genes. Our knockout of acylsugar biosynthesis is an important step toward 
making the already excellent $N$. benthamiana model system (Goodin et al., 2008; Bally et al., 2018) more suitable for studying plant-insect interactions. Future plant-insect interactions research using asat 2 mutant plants may include: (i) functional analysis of additional $N$. benthamiana genes in the asat 2 mutant background by CRISPR/Cas9 mutagenesis using a newly developed virus-mediated gRNA delivery system (Ellison et al., 2020), (ii) transient expression assays to test the function of both insect-produced elicitors and insect-defensive genes from other plant species in N. benthamiana asat2 mutants (Bos et al., 2010; Casteel et al., 2014; Elzinga et al., 2014; Rodriguez et al., 2014), and (iii) virus-induced gene silencing (VIGS) to down-regulate gene expression in insects feeding on $N$. benthamiana (Feng and Jander, 2021). Furthermore, the almost complete absence of acylsugars in the asat 2 mutant lines, coupled with the facile Agrobacterium and virus-mediated transient gene expression systems that are available for $N$. benthamiana, will make these mutants a suitable platform for the functional analysis of ASATs from other Solanaceae.

\section{Materials and Methods} Insect and plant cultures

A tobacco-adapted red strain of $M$. persicae (Ramsey et al., 2007; Ramsey et al., 2014) was maintained on $N$. tabacum plants in a growth room at $23^{\circ} \mathrm{C}$ with a $16: 8 \mathrm{~h}$ light:dark photoperiod. A colony of $B$. tabaci MEAM1 were provided by Jane Polston (University of Florida) and was maintained on tobacco (Nicotiana tabacum). Macrosiphum euphorbiae was obtained from Isgouhi Kaloshian (UC Riverside) and was maintained on tomato (Solanum lycopersicum) cv. Moneymaker. Eggs of H. zea, H. virescens, and T. $n i$ were purchased from Benzon Research (www.benzonresearch.com). Nicotiana benthamiana wild type and mutant plants for aphid experiments, caterpillar experiments were maintained at $23^{\circ} \mathrm{C}$ and a $16: 8 \mathrm{~h}$ light:dark photoperiod in a Conviron (Winnipeg, Canada) growth chamber and, for seed production, in a greenhouse at $27 / 24^{\circ} \mathrm{C}$ (day/night) with ambient light conditions. Wild type and mutant $N$. benthamiana

\section{Identification of ASAT1 and ASAT2 orthologs in N. benthamiana}

To identify ASAT1 and ASAT2 orthologs in N. benthamiana, protein sequences of Salpiglossis sinuata and Solanum lycopersicum ASAT1 and ASAT2 (Moghe et al., 2017) were compared to predicted proteins encoded by the $N$. benthamiana genome. Sequences with $>67 \%$ identity were selected as potential plants for whitefly choice and no-choice assays were maintained at $26^{\circ} \mathrm{C}$ and a $16: 8 \mathrm{~h}$ light:dark photoperiod in a growth room. ASAT1 and ASAT2 candidates and nucleotide sequences were obtained from the Solanaceae Genomics Network (www.solgenomics.net). The candidate ASAT sequences were subsequently confirmed by comparison to the most recent published N. benthamiana genome assembly (Schiavinato et al., 2019). To 
verify the nucleotide sequences of $N$. benthamiana ASAT1 and ASAT2, the genes were amplified with ASAT1F/ASAT1R and ASAT2F/ASAT2R primers (Table S4) from genomic DNA. Amplified fragments were cloned in pDONOR ${ }^{\mathrm{TM}} 207$ (ThermoFisher Scientific, US) and were sequenced in their entirety using Sanger sequencing, which showed no differences to the published N. benthamiana genome.

Phylogenetic analysis of $\mathrm{N}$. benthamiana ASATs

A protein phylogenetic tree of previously annotated Solanaceae ASATs (Fig. 2, S1, S2; Tables S1, S2) was constructed using maximum likelihood method. Briefly, the ASAT protein sequences were aligned in ClustalW (Thompson et al., 1994). Then the alignment was improved by removing the spurious sequences and poorly aligned regions (gap threshold at 0.25) using TrimAL v1.4 (Capella-Gutierrez et al., 2009). Finally, an unrooted maximum likelihood tree was generated using the improved alignment with a bootstrap of 1000 in RAxML v8.2.12 (Stamatakis, 2014). The tree was visualized and presented using FigTree v1.4.4 (http://tree.bio.ed.ac.uk).

sgRNA design and plasmid cloning

Single-guide RNAs (sgRNA) targeting ASAT1 and ASAT2 were designed based on the coding regions using CRISPR-P v2.0 (Liu et al., 2017) and CRISPRdirect (https://crispr.dbcls.jp/), based on cleavage efficiency and lack of potential off-target sites in the $N$. benthamiana genome. Additionally, only sgRNAs with $>40 \%$ GC content were selected.

Three Cas9/gRNA constructs each were constructed for ASAT1 and ASAT2 following a previously developed CRISPR/Cas9 system (Jacobs et al., 2015). Four segments of DNA were prepared with 20-bp overlaps on their ends. 1) ssDNA gRNA oligonucleotides targeting either the sense or antisense sequence of target genes were designed as: sense oligo TCAAGCGAACCAGTAGGCTTGN19-GTTTTAGAGCTAGAAATAGC, and antisense oligo GCTATTTCTAGCTCTAAAAC-N19CAAGCCTACTGGTTCGCTTGA (the gRNA sequences are shown in Fig. 3; Table S4), and synthesized by Integrated DNA Technologies (www.idtdna.com). One $\mu \mathrm{l}$ of each $100 \mu \mathrm{M}$ oligo was added to $500 \mu \mathrm{l}$ 1x NEB buffer 2 (New England Biolabs, www.neb.com). 2) The p201N:Cas9 plasmid was linearized by digestion with Spe 1 (www.neb.com) in $1 \mathrm{x}$ buffer 4 at $37^{\circ} \mathrm{C}$ for $2 \mathrm{~h}$, followed by column purification and a second digestion with $S w a l$ in $1 \mathrm{x}$ buffer 3.1 at $25^{\circ} \mathrm{C}$ for $2 \mathrm{~h}$. Complete plasmid digestion was confirmed on a $0.8 \%$ agarose gel. 3) The MtU6 promoter and 4) Scaffold DNAs were PCR-amplified from the pUC gRNA Shuttle plasmid (Jacobs et al., 2015) using the primers Swal_MtU6F/MtU6R and ScaffoldF/Spe_ScaffoldR, respectively (Table S4). The PCR reactions were performed with a highfidelity polymerase (2xKapa master mix; www.sigmaaldrich.com) using the program: $95^{\circ} \mathrm{C}$ for $3 \mathrm{~min}$ followed by $31 \mathrm{cycles}$ of $98^{\circ} \mathrm{C}$ for $20 \mathrm{sec}, 60^{\circ} \mathrm{C}$ for $30 \mathrm{sec}, 72^{\circ} \mathrm{C}$ for $30 \mathrm{sec}$, and a final extension of $72^{\circ} \mathrm{C}$ 
for 5 min. Finally, cloning was done using the NEBuilder ${ }^{\circledR}$ HiFi DNA Assembly Cloning Kit. For each reaction, the four pieces of DNA were mixed in a $20-\mu 1$ reaction with the NEBbuilder assembly mix with a final concentration of $0.011 \mathrm{pmol}(\sim 100 \mathrm{ng})$ of p201N:Cas9 plasmid, $0.2 \mathrm{pmol}$ of MtU6 amplicon ( $\sim 50$ ng), scaffold amplicon ( 12 ng) and ssDNA gRNA oligo (60-mer, $1 \mu \mathrm{l})$. The reactions were incubated at $50^{\circ} \mathrm{C}$ for $1 \mathrm{~h}$.

Two $\mu$ l of the cloning reaction were transformed into $50 \mu 1$ of One Shot ${ }^{\mathrm{TM}}$ Top 10 chemically competent cells (Invitrogen, www.thermofisher.com) and plated on LB (Bertani, 1951) agar medium with benthamiana genome v1.0.1 (www.solgenomics.net). Only two sites were found to have non-target matches $>17 \mathrm{nt}$ (both with 1 internal mismatch), and with the NGG PAM sequence on the correct strand. These two sites were checked by PCR amplification and Sanger sequencing (primers in Table S4) and showed no unexpected editing in our ASAT mutant plants.

Stable mutagenesis of ASATs using tissue culture

Stable ASAT mutant $N$. benthamiana plants were created in the Boyce Thompson Institute plant transformation facility using CRISPR/Cas9 with gRNAs that had been confirmed to be functional in transient assays (Fig. S9) following a previously described protocol (Van Eck et al., 2019), with minor modifications.

\section{Confirmation of homozygous mutant plants in the T2 generation}

Rooted $N$. benthamiana plants from tissue culture were transferred to soil (T0 generation). CRISPR/Cas9induced mutations were identified by PCR amplification of genomic regions of the gRNA target sites in ASAT1 and ASAT2 (Fig. 3), followed by Sanger sequencing (primers in Table S4). Lines with mutations were used to generate T1 plants, which were subjected to PCR amplification and sequencing to confirm homozygous mutations. T2 seeds from confirmed homozygous mutant asat1-1, asat1-2, asat2-1, and asat2-2 T1 plants were used for all experiments. Homozygous mutations were confirmed in randomly selected T2 plants by PCR amplification and Sanger sequencing. The presence or absence of Cas9 in transgenic plants in the T0, T1, and T2 generations was confirmed by PCR amplification (primers in 
433 Liquid chromatography/mass spectrometry (LC/MS) was used to measure acylsugar content in leaf

434 extracts from wildtype and ASAT mutant plants. New leaflets were rinsed in acylsugar extraction solution

435 (3:3:2 acetonitrile:isopropanol:water, $0.1 \%$ formic acid, and $1 \mu \mathrm{M}$ Telmisartan as internal standard) and

436 gently agitated for $2 \mathrm{~min}$. Then, the extraction solutions were transferred to LC/MS glass vials, and the

437 leaves were air dried for leaf weight measurements.

438 Chromatography of leaf surface washes was performed on a ThermoScientific Ultimate 3000

439 HPLC with a glass vial autosampler coupled with a Thermo Scientific Q Exactive ${ }^{\mathrm{TM}}$ Hybrid Quadrupole-

440 Orbitrap ${ }^{\mathrm{TM}}$ Mass Spectrometer at Boyce Thompson Institute. Acylsugar extracts were separated on an

441 Ascentis Express C18 HPLC column $(10 \mathrm{~cm} \times 2.1 \mathrm{~mm} \times 2.7 \mu \mathrm{m})($ Sigma-Aldrich, St. Louis, MO) with a

442 flow rate of $0.3 \mathrm{ml} / \mathrm{min}$, using a gradient flow of $0.1 \%$ formic acid (Solvent A) and $100 \%$ acetonitrile

443 (Solvent B). We used a 7-min LC method for metabolite profiling, which involved a linear gradient from

444 95:5 A:B to 0:98 A:B. Full-scan mass spectra were collected (mass range: $\mathrm{m} / \mathrm{z}$ 50-1000) in both positive

445 and negative electron spray ionization (ESI) modes. Mass spectral parameters were set as follows:

446 capillary spray voltage $2.00 \mathrm{kV}$ for negative ion-mode and $3.00 \mathrm{kV}$ for positive ion-mode, source

447 temperature: $100^{\circ} \mathrm{C}$, desolvation temperature $350^{\circ} \mathrm{C}$, desolvation nitrogen gas flow rate: 600 liters/h, cone

448 voltage 35 V. Acylsugars were identified and annotated using Thermo Xcalibur Qual Browser (Thermo

449 Fisher) and MS-DIAL v4.20 based on the MS/MS peak features and neutral losses (Fig. S3). The

450 acylsugar abundances were estimated using peak areas at the respective $m / z$ channel under negative ESI

451 mode. Acylsugar quantification was first normalized to the internal control Telmisartan to account for

452 technical variation, and then normalized to the leaf dry weight to allow comparisons between samples.

454 Insect choice and no-choice bioassays

455 To measure $M$. persicae and M. euphorbiae growth, we caged aphids on individual leaves of mutant and

456 wildtype 4 5-week-old N. benthamiana (Fig. S10a,b). Twenty adult M. persicae from N. tabacum (naïve

457 to $N$. benthamiana) were placed in each cage and allowed to generate nymphs for $\sim 12 \mathrm{hrs}$. Twenty-five

458 nymphs were left in each cage and were monitored for $5 \mathrm{~d}$ to assess nymph survival. At the end of the

459 survival monitoring period, five $M$. persicae were left in each cage and reproduction was monitored for

460 one week. The remaining $M$. persicae were collected to measure aphid size by imaging and assessing the

461 area of each aphid using ImageJ (Schneider et al., 2012). Ten adult M. euphorbiae from a colony on

462 tomato cv. Moneymaker were placed in each individual cage on N. benthamiana leaves. Surviving aphids

463 and progeny were counted after $24 \mathrm{~h}$.

464 M. persicae and M. euphorbiae choice assays were performed with detached leaves from 4 5-

465 week-old N. benthamiana. Two similarly-sized leaves from individual ASAT mutant and wildtype plants 
were cut and placed in 15-cm Petri dishes, with their petioles inserted in moistened cotton swabs (Fig. S10c). Ten naïve adult aphids were released at the midpoint between pairs of leaves (wildtype, asatl, or asat2), and the Petri dishes were placed under 16:8 h light:dark photoperiod. The aphids on each leaf were counted at $24 \mathrm{~h}$ after their release in the Petri dishes.

To measure whitefly survival and fecundity on wildtype and asat2-1 N. benthamiana plants, cages were set up with plants at the seven-leaf stage ( $\sim 3$ weeks old). Each cage contained three plants,

472 either wildtype or asat2-1. Ninety adult whiteflies reared on Brassica oleracea (variety Earliana;

473 www.burpee.com, catalog\# 62729A) were introduced into each cage $(60 \times 60 \times 60 \mathrm{~cm})$ with $N$.

474 benthamiana (30 whiteflies/plant) and were allowed to feed for three days at $26^{\circ} \mathrm{C}$ with a $16: 8 \mathrm{~h}$

475 light:dark photoperiod. The numbers of whiteflies surviving on each host plant were counted, after which

476 the remaining insects were killed with insecticidal soap. The following day, the number of whitefly eggs on each plant was counted. This experiment was conducted twice with similar results.

For whitefly choice assays, wildtype and asat2-1 plants at the seven-leaf stage were placed together in the same cage. Approximately 150 whiteflies from cabbage plants were moved into each cage. After $24 \mathrm{~h}$ at $26^{\circ} \mathrm{C}$ with a 16:8 $\mathrm{h}$ light:dark photoperiod, live and dead whiteflies were counted on the plants and elsewhere in the cage. This experiment was repeated three times.

Eggs of H. zea, H. virescens and T. ni were hatched on artificial diet (Southland Products, Lake Village, Arkansas). Neonate larvae were confined onto individual N. benthamiana leaves, one larva/plant, using 10 x $15 \mathrm{~cm}$ organza mesh bags (www.amazon.com, item B073J4RS9C). After ten days, the surviving larvae were counted and weighed. In a separate experiment, H. zea, H. virescens, and T. ni were reared on artificial diet (beet armyworm diet, www.southlandproducts.net) for five days. Individual fiveday-old caterpillars were weighed and then confined on 4 4.5-week-old $N$ benthamiana plants using 30 $\mathrm{cm}$ x $60 \mathrm{~cm}$ micro-perforated bread bags (www.amazon.com). After seven days, the surviving larvae were weighed again. Relative growth rate was calculated as: $\ln ((($ day-12 mass $) /($ mean day-5 mass $)) / 7)$.

\section{Leaf water loss and temperature assays}

492 To measure the leaf water loss, two leaves from each of eight plants were detached. The fresh weight of

493 each leaf was determined on a Sartorius Ultra Micro Balance. All leaves were placed at $23^{\circ} \mathrm{C}$ and a $16: 8 \mathrm{~h}$

494 light:dark photoperiod. Each leaf was weighted again after $24 \mathrm{~h}$ and the percentage of water loss was

495 calculated as [(fresh_weight - final_weight)/fresh_weight]*100\%.

496 Thermal images were acquired in the growth chamber using a thermal camera (A655sc, FLIR

497 Systems Inc., Boston, MA) with a spectral range of 7.5-14.0 mm and a resolution of $640 \mathrm{x} 480$ pixels.

498 The camera was placed $\sim 1 \mathrm{~m}$ away from each plant and a white background was used when the plant

499 images were acquired. One region of interest (ROI), corresponding to the perimeter of each leaf, was 
specified per leaf for 20 leaves per genotype. Using the FLIR ResearchIR Max software v.4.40.9.30, thermal images files were exported as CSV files. Images were segmented from the background using Gaussian mixture models in MATLAB to determine the temperature of each leaf. After segmentation, the temperature was averaged across the segmented leaf.

Hyperspectral images were acquired in a dark room using a hyperspectral imager (SOC710, Series 70-V, Surface Optics Corporation, San Diego, CA) that covered a 400-1000 nm spectral range for 128 wavebands. Image acquisition were performed using a Dell DELL XPS 159570 laptop computer that controls the camera. The camera was fixed $\sim 1 \mathrm{~m}$ above the plants and capturing top view images. A Spectralon tile (Labsphere Inc, North Sutton, NH) was placed next to the plant trays, covering one corner of the image to facilitate subsequent image processing and calibration. The nominal reflectance value for the Spectralon tile was $99 \%$ with a $30.5 \times 30.5 \mathrm{~cm}^{2}$ reflective area. Lighting consisted of two halogen lamps placed at $\sim 45^{\circ}$ angles on either side of the camera to create an even light distribution. All image analysis was performed in HSIviewer, a MATLAB package (Stone et al., 2020). White reflectance calibration was performed using the Spectralon tile. One ROI was specified for each of 20 leaves per genotype. This ROI corresponded to the perimeter of each leaf. From each hyperspectral cube image, the vegetation pixels (green portion of the plant) were extracted using the Normalized Difference Vegetation Index (NDVI). Mean reflectance (R) was calculated per band per 10 leaves in order to obtain the water band index (WBI) results. To calculate NDVI and WBI we used the following formulas, where $R$ corresponds to the reflectance at a specific wavelength (nm): WBI $=($ R970/R900) (Penuelas et al., 1993) and NVDI = (R750 - R705)/(R750 + R705) (Gitelson and Merzlyak, 1994).

\section{Statistical analysis}

522 All statistical comparisons were conducted using SPSS v25, R and MATLAB R2019a (MathWorks, Inc., 523 Natick, MA, USA). ANOVA followed by a Dunnett's post hoc test was used to determine differences in

524 leaf water loss, leaf temperature, and WBI across genotypes in each data set. ANOVA followed by a

525 Duncan post hoc test was used for aphid bioassay and LC/MS results. A Chi-square test was used to test

526 for differences in pairwise aphid choice assays, whitefly, and lepidopteran assays. Raw data for all figures 527 are shown in Table S3.

\section{Declarations:}

\section{Acknowledgements}

531 We thank Patricia Keen and Joyce Van Eck for their help with N. benthamiana stable transformation,

532 Ning Zhang and Greg Martin for sharing the p201N-Cas9 construct and the Drm3 sgRNA control

533 constructs, and William Stone and Thomas Lawton for providing custom image processing software. 


\section{Funding}

536 This research was supported by Cornell startup funds to G.D.M., Deutsche Forschungsgemeinschaft

537 award \#411255989 to L.H.K., and United States Department of Agriculture Biotechnology Risk

538 Assessment Grant 2017-33522-27006, US National Science Foundation award IOS-1645256, and

539 Defense Advanced Research Projects Agency (DARPA) agreement HR0011-17-2-0053 to G.J, and US

540 National Science Foundation award \#1723926 to C.L.C. G.S. is part of a team supporting DARPA's

541 Insect Allies program under agreement HR0011-17-2-0055. M.A.G. is part of a team supporting

542 DARPA's Advanced Plant Technologies program under agreement HR0011-18-C-0146. The views and

543 conclusions contained in this document are those of the authors and should not be interpreted as

544 representing the official policies of the U.S. Government.

\section{Conflict of interest}

547 The authors declare that there is no conflict of interest.

\section{Availability of data and material}

550 All data generated or analyzed during this study are included in this published article and its 551 supplementary information files.

\section{Code availability}

554 Not applicable

\section{Authors' Contributions}

557 G.J. and H.F. conceived the original research plans; H.F., S.S., L.A., H.X., L.K., J.D.T, S.H.C., and 558 A.N.F. performed the experiments; H.F., L.A., L.K., and G.D.M. analyzed the data; C.L.C., M.A.G., 559 G.D.M., G.S., and G.J. supervised the experiments; H.F. and G.J. wrote the article with contributions 560 from all of the authors; G.J. agrees to serve as the contact author responsible for communication and 561 distribution of samples.

\section{Ethics approval}

564 Not applicable

\section{Consent to Participate}

567 Not applicable 


\section{Consent for publication}

570 Not applicable

\section{References}

573 Arntzen C (2015) Plant-made pharmaceuticals: from 'Edible Vaccines' to Ebola therapeutics. Plant Biotechnology Journal 13: 1013-1016

Arrendale RF, Severson RF, Sisson VA, Costello CE, Leary JA, Himmelsbach DS, Vanhalbeek H (1990) Characterization of the sucrose ester fraction from Nicotiana glutinosa. Journal of Agricultural and Food Chemistry 38: 75-85

Bally J, Jung H, Mortimer C, Naim F, Philips JG, Hellens R, Bombarely A, Goodin MM, Waterhouse PM (2018) The rise and rise of Nicotiana benthamiana: a plant for all reasons. Annual Review of Phytopathology, Vol 56 56: 405-426

Bertani G (1951) Studies on lysogenesis. I. The mode of phage liberation by lysogenic Escherichia coli. J Bacteriol 62: 293-300

Bombarely A, Rosli HG, Vrebalov J, Moffett P, Mueller LA, Martin GB (2012) A draft genome sequence of Nicotiana benthamiana to enhance molecular plant-microbe biology research. Mol Plant-Micr Int 25: 1523-1530

Bos JI, Prince D, Pitino M, Maffei ME, Win J, Hogenhout SA (2010) A functional genomics approach identifies candidate effectors from the aphid species Myzus persicae (green peach aphid). PLoS Genet 6: e1001216

Capella-Gutierrez S, Silla-Martinez JM, Gabaldon T (2009) trimAl: a tool for automated alignment trimming in large-scale phylogenetic analyses. Bioinformatics 25: 1972-1973

Cardoso MZ (2008) Herbivore handling of a plant's trichome: The case of Heliconius charithonia (L.) (Lepidoptera : Nymphalidae) and Passiflora Lobata (Killip) Hutch. (Passifloraceae). Neotropical Entomology 37: 247-252

Casteel CL, Yang C, Nanduri AC, De Jong HN, Whitham SA, Jander G (2014) The NIa-Pro protein of turnip mosaic virus improves growth and reproduction of the aphid vector, Myzus persicae (green peach aphid). Plant J 77: 653-663

Chortyk OT, Pomonis JG, Johnson AW (1996) Syntheses and characterizations of insecticidal sucrose esters. J Ag Food Chem 44: 1551-1557

Clay N, Adio A, Denoux C, Jander G, Ausubel F (2009) Glucosinolate metabolites required for an Arabidopsis innate immune response. Science 323: 95-101

D'Auria JC (2006) Acyltransferases in plants: a good time to be BAHD. Curr Opin Plant Biol 9: 331-340

Egan AN, Moore S, Stellari GM, Kang BC, Jahn MM (2019) Tandem gene duplication and recombination at the AT3 locus in the Solanaceae, a gene essential for capsaicinoid biosynthesis in Capsicum. Plos One 14: e0210510

Egea I, Albaladejo I, Meco V, Morales B, Sevilla A, Bolarin MC, Flores FB (2018) The drought-tolerant Solanum pennellii regulates leaf water loss and induces genes involved in amino acid and ethylene/jasmonate metabolism under dehydration. Sci Rep 8: 2791

Ellison EE, Nagalakshmi U, Gamo ME, Huang PJ, Dinesh-Kumar S, Voytas DF (2020) Multiplexed heritable gene editing using RNA viruses and mobile single guide RNAs. Nat Plants 6: 620-624

Elzinga DA, De Vos M, Jander G (2014) Suppression of plant defenses by a Myzus persicae (green peach aphid) salivary effector protein. Mol Plant Microbe Interact 27: 747-756

Fan P, Miller AM, Schilmiller AL, Liu X, Ofner I, Jones AD, Zamir D, Last RL (2016) In vitro reconstruction and analysis of evolutionary variation of the tomato acylsucrose metabolic

614 network. Proc Natl Acad Sci U S A 113: E239-248 
615

616

617

618

619

620

621

622

623

624

625

626

627

628

629

630

631

632

633

634

635

636

637

638

639

640

641

642

643

644

645

646

647

648

649

650

651

652

653

654

655

656

657

658

659

660

661

662

663

664

665

Feng H, Jander G (2021) Rapid screening of Myzus persicae (green peach aphid) RNAi targets using Tobacco rattle virus. Methods Mol Biol in press

Fobes JF, Mudd JB, Marsden MP (1985) Epicuticular lipid accumulation on the leaves of Lycopersicon pennellii (Corr.) D'Arcy and Lycopersicon esculentum Mill. Plant Physiol 77: 567-570

Gaquerel E, Kotkar H, Onkokesung N, Galis I, Baldwin IT (2013) Silencing an N-acyltransferase-like involved in lignin biosynthesis in Nicotiana attenuata dramatically alters herbivory-induced phenolamide metabolism. Plos One 8: e62336

Gitelson A, Merzlyak MN (1994) Spectral reflectance changes associated with autumn senescence of Aesculus hippocastanum $\mathrm{L}$ and Acer platanoides $\mathrm{L}$ leaves - spectral features and relation to chlorophyll estimation. J Plant Physiol 143: 286-292

Glas JJ, Schimmel BCJ, Alba JM, Escobar-Bravo R, Schuurink RC, Kant MR (2012) Plant glandular trichomes as targets for breeding or engineering of resistance to herbivores. International Journal of Molecular Sciences 13: 17077-17103

Gong P, Zhang J, Li H, Yang C, Zhang C, Zhang X, Khurram Z, Zhang Y, Wang T, Fei Z, Ye Z (2010) Transcriptional profiles of drought-responsive genes in modulating transcription signal transduction, and biochemical pathways in tomato. J Exp Bot 61: 3563-3575

Goodin MM, Zaitlin D, Naidu RA, Lommel SA (2008) Nicotiana benthamiana: its history and future as a model for plant-pathogen interactions. Mol Plant Microbe Interact 21: 1015-1026

Hagimori M, Matsui M, Matsuzaki T, Shinozaki Y, Shinoda T, Harada H (1993) Production of somatic hybrids between Nicotiana benthamiana and Nicotiana tabacum and their resistance to aphids. Plant Science 91: 213-222

Jacobs TB, LaFayette PR, Schmitz RJ, Parrott WA (2015) Targeted genome modifications in soybean with CRISPR/Cas9. Bmc Biotechnology 15: 16

Karabourniotis G, Kotsabassidis D, Manetas Y (1995) Trichome density and its protective potential against ultraviolet-B radiation damage during leaf development. Canadian Journal of BotanyRevue Canadienne De Botanique 73: 376-383

Kim J, Kang K, Gonzales-Vigil E, Shi F, Jones AD, Barry CS, Last RL (2012) Striking natural diversity in glandular trichome acylsugar composition is shaped by variation at the acyltransferase 2 locus in the wild tomato Solanum habrochaites. Plant Physiol 160: 1854-1870

Kroumova AB, Wagner GJ (2003) Different elongation pathways in the biosynthesis of acyl groups of trichome exudate sugar esters from various solanaceous plants. Planta 216: 1013-1021

Kroumova ABM, Zaitlin D, Wagner GJ (2016) Natural variability in acyl moieties of sugar esters produced by certain tobacco and other Solanaceae species. Phytochemistry 130: 218-227

Laue G, Preston CA, Baldwin IT (2000) Fast track to the trichome: induction of N-acyl nornicotines precedes nicotine induction in Nicotiana repanda. Planta 210: 510-514

Leong BJ, Lybrand DB, Lou YR, Fan PX, Schilmiller AL, Last RL (2019) Evolution of metabolic novelty: A trichome-expressed invertase creates specialized metabolic diversity in wild tomato. Science Advances 5: eaaw3754

Liu H, Ding YD, Zhou YQ, Jin WQ, Xie KB, Chen LL (2017) CRISPR-P 2.0: an improved CRISPRCas9 tool for genome editing in plants. Molecular Plant 10: 530-532

Marchant WG, Legarrea S, Smeda JR, Mutschler MA, Srinivasan R (2020) Evaluating acylsugarsmediated resistance in tomato against Bemisia tabaci and transmission of tomato yyellow leaf curl virus. Insects 11: 842

Matsuzaki T, Shinozaki Y, Hagimori M, Tobita T, Shigematsu H, Koiwai A (1992) Novel glycerolipids and glycolipids from the surface-lipids of Nicotiana benthamiana. Bioscience Biotechnology and Biochemistry 56: 1565-1569

Matsuzaki T, Shinozaki Y, Suhara S, Ninomiya M, Shigematsu H, Koiwai A (1989) Isolation of glycolipids from the surface-lipids of Nicotiana bigelovii and their distribution in Nicotiana species. Agricultural and Biological Chemistry 53: 3079-3082

McKenzie CL, Puterka GJ (2004) Effect of sucrose octanoate on survival of nymphal and adult Diaphorina citri (Homoptera : Psyllidae). J Econ Entomol 97: 970-975 
666

667

668

669

670

671

672

673

674

675

676

677

678

679

680

681

682

683

684

685

686

687

688

689

690

691

692

693

694

695

696

697

698

699

700

701

702

703

704

705

706

707

708

709

710

711

712

713

714

715

McKenzie CL, Weathersbee AA, 3rd, Puterka GJ (2005) Toxicity of sucrose octanoate to egg, nymphal, and adult Bemisia tabaci (Hemiptera: Aleyrodidae) using a novel plant-based bioassay. J Econ Entomol 98: 1242-1247

Meihls LN, Handrick V, Glauser G, Barbier H, Kaur H, Haribal MM, Lipka AE, Gershenzon J, Buckler ES, Erb M, Köllner TG, Jander G (2013) Natural variation in maize aphid resistance is associated with 2,4-dihydroxy-7-methoxy-1,4-benzoxazin-3-one glucoside methyltransferase activity. Plant Cell 25: 2341-2355

Moghe GD, Leong BJ, Hurney SM, Jones AD, Last RL (2017) Evolutionary routes to biochemical innovation revealed by integrative analysis of a plant-defense related specialized metabolic pathway. Elife 6: e28468

Ning J, Moghe GD, Leong B, Kim J, Ofner I, Wang Z, Adams C, Jones AD, Zamir D, Last RL (2015) A feedback-insensitive isopropylmalate synthase affects acylsugar composition in cultivated and wild tomato. Plant Physiol 169: 1821-1835

O' Connell MA, Medina AL, Sanchez Pena Pand Trevino MB (2007) Molecular genetics of drought resistance response in tomato and related species. In Razdan, M. K., \& Mattoo, A. K. (eds). Genetic Improvement of Solanaceous Crops 2: Enfield USA 261-283

Penuelas J, Filella I, Biel C, Serrano L, Save R (1993) The reflectance at the 950-970 $\mathrm{nm}$ region as an indicator of plant water status. International Journal of Remote Sensing 14: 1887-1905

Powell JD (2015) From pandemic preparedness to biofuel production: tobacco finds its biotechnology niche in North America. Agriculture-Basel 5: 901-917

Ramsey JS, Elzinga DA, Sarkar P, Xin YR, Ghanim M, Jander G (2014) Adaptation to nicotine feeding in Myzus persicae. J Chem Ecol 40: 869-877

Ramsey JS, Wilson AC, De Vos M, Sun Q, Tamborindeguy C, Winfield A, Malloch G, Smith DM, Fenton B, Gray SM, Jander G (2007) Genomic resources for Myzus persicae: EST sequencing, SNP identification, and microarray design. BMC Genomics 8: 423

Rodriguez AE, Tingey WM, Mutschler MA (1993) Acylsugars of Lycopersicon pennellii deter settling and feeding of the green peach aphid (Homoptera, Aphididae). Journal of Economic Entomology 86: $34-39$

Rodriguez PA, Stam R, Warbroek T, Bos JI (2014) Mp10 and Mp42 from the aphid species Myzus persicae trigger plant defenses in Nicotiana benthamiana through different activities. Mol Plant Microbe Interact 27: 30-39

Schiavinato M, Strasser R, Mach L, Dohm JC, Himmelbauer H (2019) Genome and transcriptome characterization of the glycoengineered Nicotiana benthamiana line DeltaXT/FT. BMC Genomics 20: 594

Schilmiller A, Shi F, Kim J, Charbonneau AL, Holmes D, Jones AD, Last RL (2010) Mass spectrometry screening reveals widespread diversity in trichome specialized metabolites of tomato chromosomal substitution lines. Plant Journal 62: 391-403

Schilmiller AL, Charbonneau AL, Last RL (2012) Identification of a BAHD acetyltransferase that produces protective acyl sugars in tomato trichomes. Proc Natl Acad Sci U S A 109: 1637716382

Schilmiller AL, Moghe GD, Fan P, Ghosh B, Ning J, Jones AD, Last RL (2015) Functionally divergent alleles and duplicated Loci encoding an acyltransferase contribute to acylsugar metabolite diversity in Solanum trichomes. Plant Cell 27: 1002-1017

Schneider CA, Rasband WS, Eliceiri KW (2012) NIH Image to ImageJ: 25 years of image analysis. Nature Methods 9: 671-675

Shepherd RW, Wagner GJ (2007) Phylloplane proteins: emerging defenses at the aerial frontline? Trends in Plant Science 12: 51-56

Simmons AT, Gurr GM, McGrath D, Martin PM, Nicol HI (2004) Entrapment of Helicoverpa armigera (Hubner) (Lepidoptera:Noctuidae) on glandular trichomes of Lycopersicon species. Australian Journal of Entomology 43: 196-200 
Simon B, Cenis JL, Demichelis S, Rapisarda C, Caciagli P, Bosco D (2003) Survey of Bemisia tabaci (Hemiptera: Aleyrodidae) biotypes in Italy with the description of a new biotype (T) from Euphorbia characias. Bull Entomol Res 93: 259-264

Slocombe SP, Schauvinhold I, McQuinn RP, Besser K, Welsby NA, Harper A, Aziz N, Li Y, Larson TR, Giovannoni J, Dixon RA, Broun P (2008) Transcriptomic and reverse genetic analyses of branched-chain fatty acid and acyl sugar production in Solanum pennellii and Nicotiana benthamiana. Plant Physiol 148: 1830-1846

Song Z, Li S, Chen X, Liu L, Song Z (2006) Synthesis of sucrose esters. Forestry Studies in China 8: 2629

Stamatakis A (2014) RAxML version 8: a tool for phylogenetic analysis and post-analysis of large phylogenies. Bioinformatics 30: 1312-1313

Stone WD, Pellicore MJ, Hagstrom S, Lawton TJ (2020) HSIviewer: Pushbutton hyperspectral image analysis for rapid plant phenotyping. in review

Thompson JD, Higgins DG, Gibson TJ (1994) Clustal-W - Improving the sensitivity of progressive multiple sequence alignment through sequence weighting, position-specific gap penalties and weight matrix choice. Nucleic Acids Research 22: 4673-4680

Thurston R (1961) Resistance in Nicotiana to the green peach aphids and some other tobacco insect pest. Journal of Economic Entomology 54: 946-949

Van Eck J, Keen P, Tjahjadi M (2019) Agrobacterium tumefaciens-mediated transformation of tomato. Methods Mol Biol 1864: 225-234

Van T, Weinhold A, Ullah C, Dressel S, Schoettner M, Gase K, Gaquerel E, Xu SQ, Baldwin IT (2017) $\mathrm{O}$-acyl sugars protect a wild tobacco from both native fungal pathogens and a specialist herbivore. Plant Physiology 174: 370-386

Wagner GJ, Wang E, Shepherd RW (2004) New approaches for studying and exploiting an old protuberance, the plant trichome. Annals of Botany 93: 3-11

Weinhold A, Baldwin IT (2011) Trichome-derived O-acyl sugars are a first meal for caterpillars that tags them for predation. Proceedings of the National Academy of Sciences of the United States of America 108: 7855-7859 


\section{Figures}
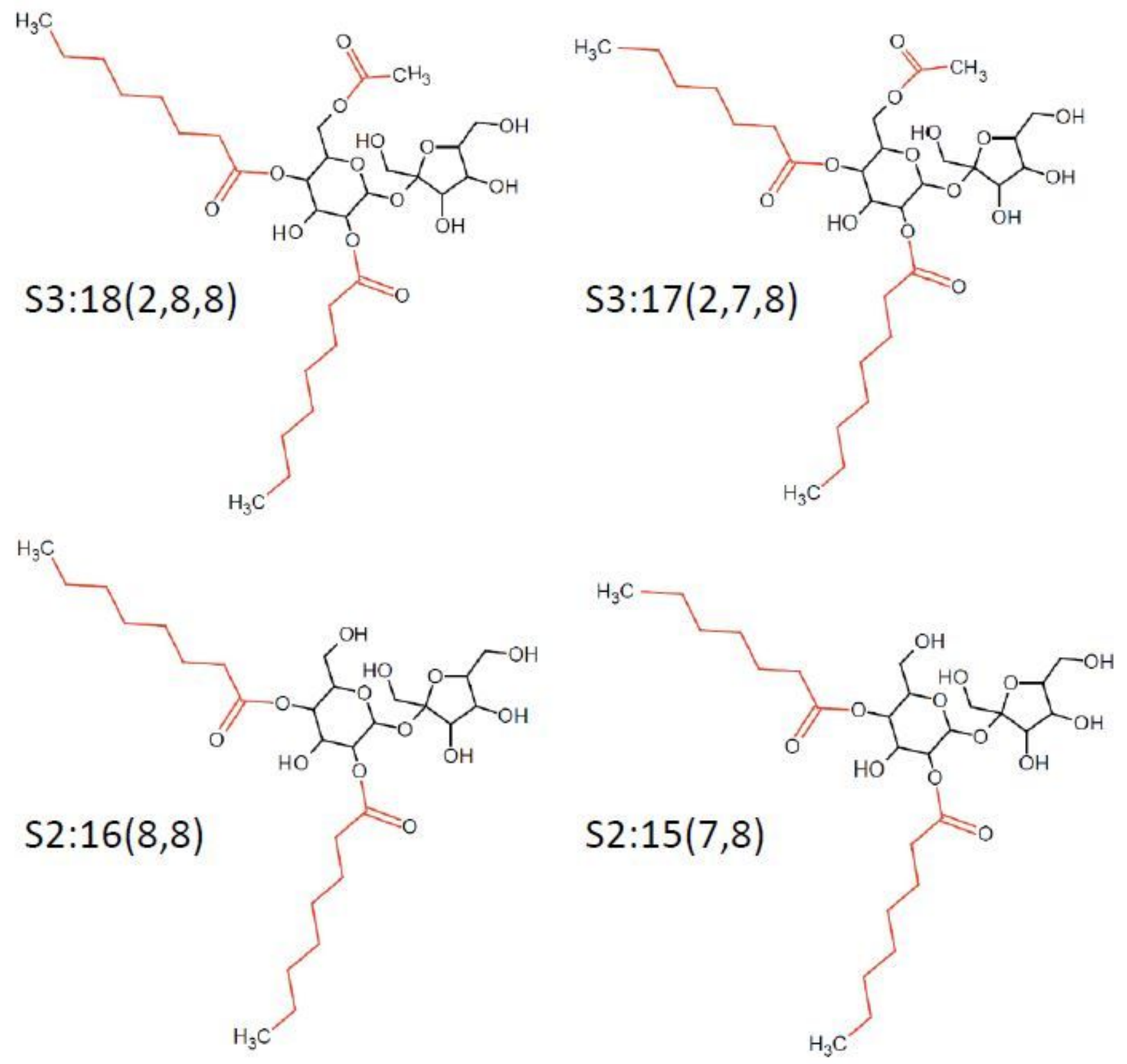

\section{Figure 1}

Predominant acylsugars in Nicotiana benthamiana Acylsucroses S3:17(2,7,8), S3:18(2,8,8), S2:16(8,8) and $\mathrm{S} 2: 15(7,8)$ are present in $\mathrm{N}$. benthamiana. In the acylsugar structure names, $\mathrm{S}$ refers to sucrose, followed by the number of acyl chains, the total length of acyl chains, and the length of each individual chain in parentheses. Although the presence of $\mathrm{C} 2, \mathrm{C} 7$, and $\mathrm{C} 8$ chain lengths is confirmed, the specific positions of the acyl chains on the sucrose molecule are hypothesized based on previous observations of acylsugars in Nicotiana alata (Moghe et al, 2017), the predicted evolution of the acylsugar biosynthetic pathway, and enzyme promiscuities in the Solanaceae family. 


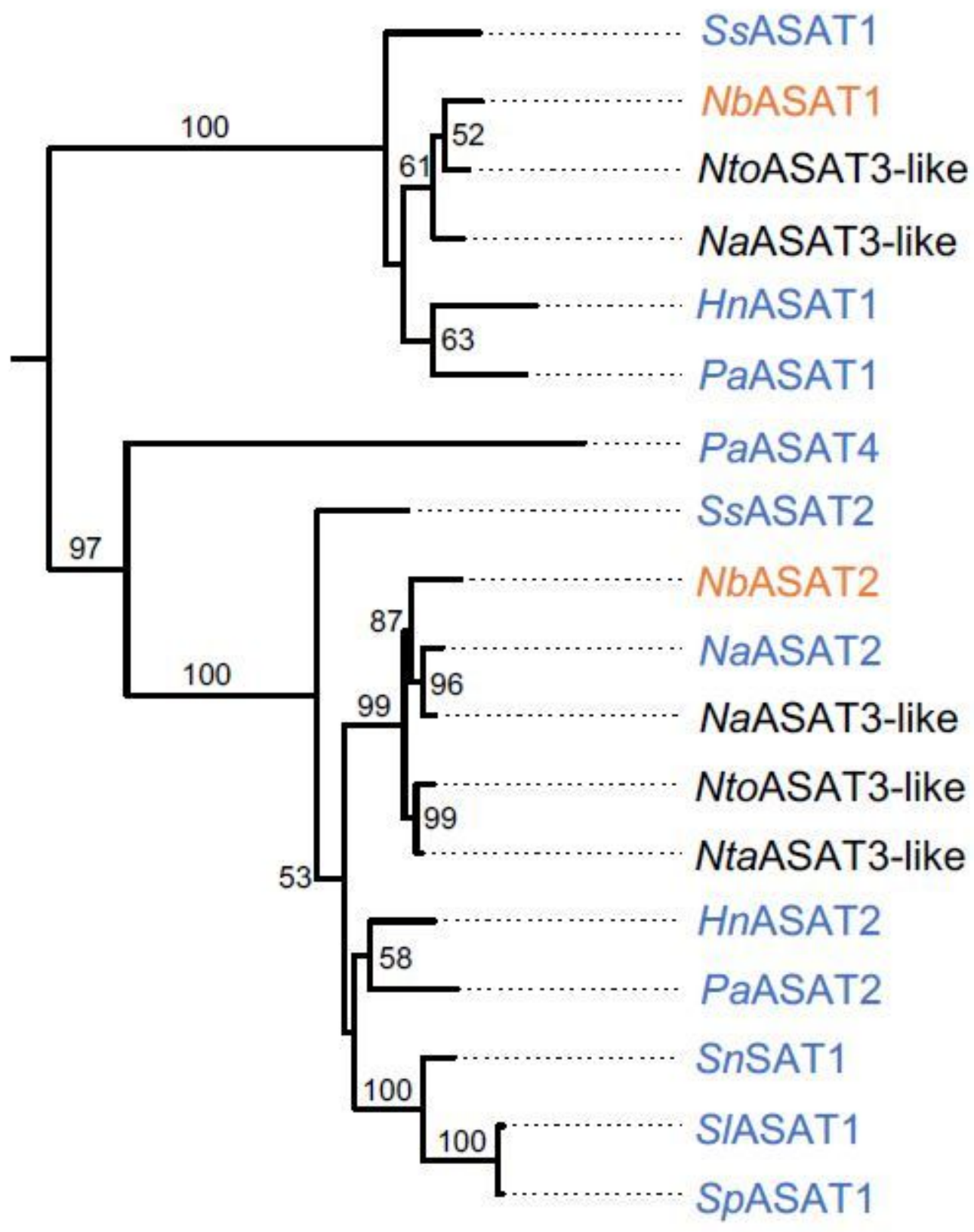

Figure 2

ASAT phylogenetic tree The evolutionary history of ASATs in the Solanaceae (Tables S1 and S2) was inferred using the Maximum Likelihood method in RAxML. Presented is a subtree of a larger tree that includes all annotated ASATs (Figure S1). The branch labels indicate the percentage of trees in which the associated taxa clustered together (bootstrap of 1000). Only values greater than 50 are presented. The two predicted N. benthamiana ASATs are highlighted in orange and ASATs that were previously chemically characterized are highlighted in blue. $\mathrm{Ss}=$ Salpiglossis sinuata, $\mathrm{Nb}=$ Nicotiana benthamiana, $\mathrm{Nto}=$ Nicotiana tomentosiformis, $\mathrm{Na}=$ Nicotiana attenuata, $\mathrm{Hn}=$ Hyoscyamus niger, $\mathrm{Pa}=$ Petunia 
axillaris, $\mathrm{Nta}=$ Nicotiana tabacum, $\mathrm{Sn}=$ Solanum nigrum, $\mathrm{SI}=$ Solanum lycopersicum, $\mathrm{Sp}=$ Solanum pennellii.
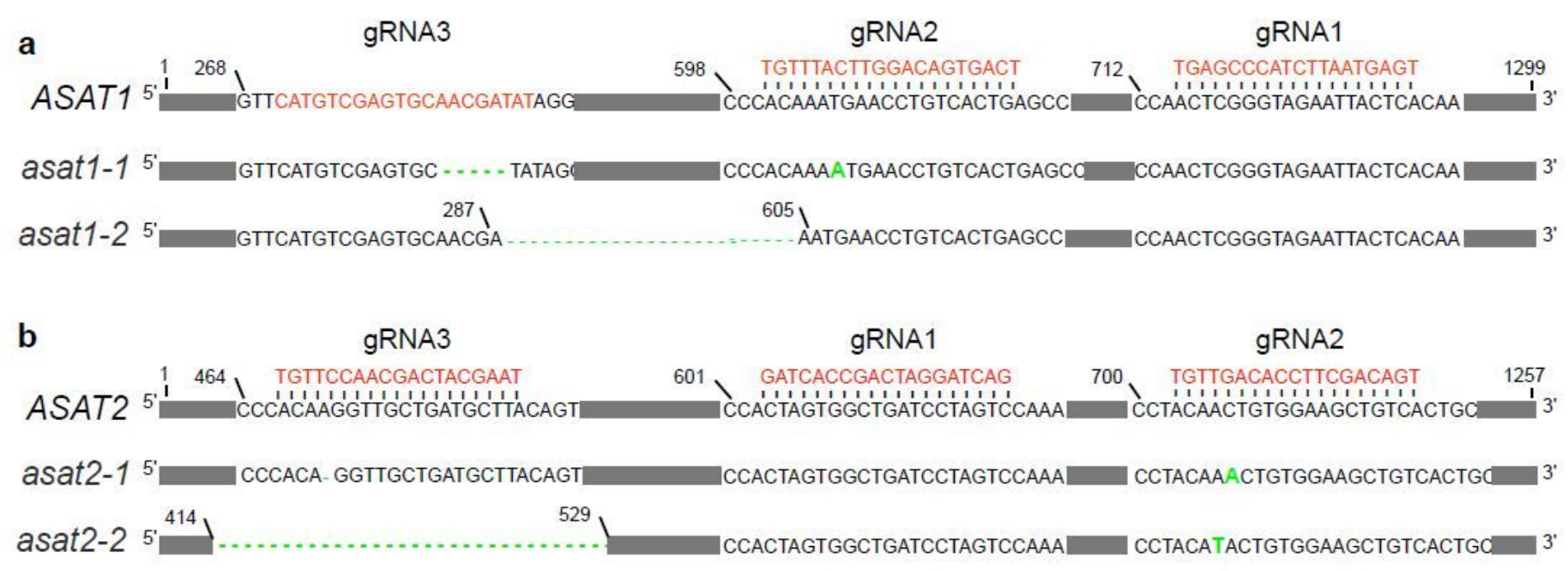

\section{Figure 3}

N. benthamiana ASAT mutations produced with CRISPR/Cas9 Three gRNAs (sequences shown in red) were designed to edit either ASAT1 or ASAT2. Whereas gRNA3 for ASAT1 is on the sense strand, the other gRNAs are on the antisense strand. For both ASAT1 and ASAT2, we obtained two independent mutations resulting from the corresponding gRNA2 and gRNA3. Single-base mutations and deletions are shown in green. (a) asat1-1 has a five-nucleotide deletion at gRNA3 and a single-nucleotide insertion at gRNA2. asat1-2 has a 318-nucleotide deletion between the gRNA3 and gRNA2 cutting sites. (b) asat2-1 has a single-nucleotide deletion at gRNA3 and single-nucleotide insertion at gRNA2 leading to a translation frame shift between the two mutations. asat2-2 has a 115-nucleotide deletion at gRNA3 and a singlenucleotide insertion leading to a translation frame shift at gRNA2. 


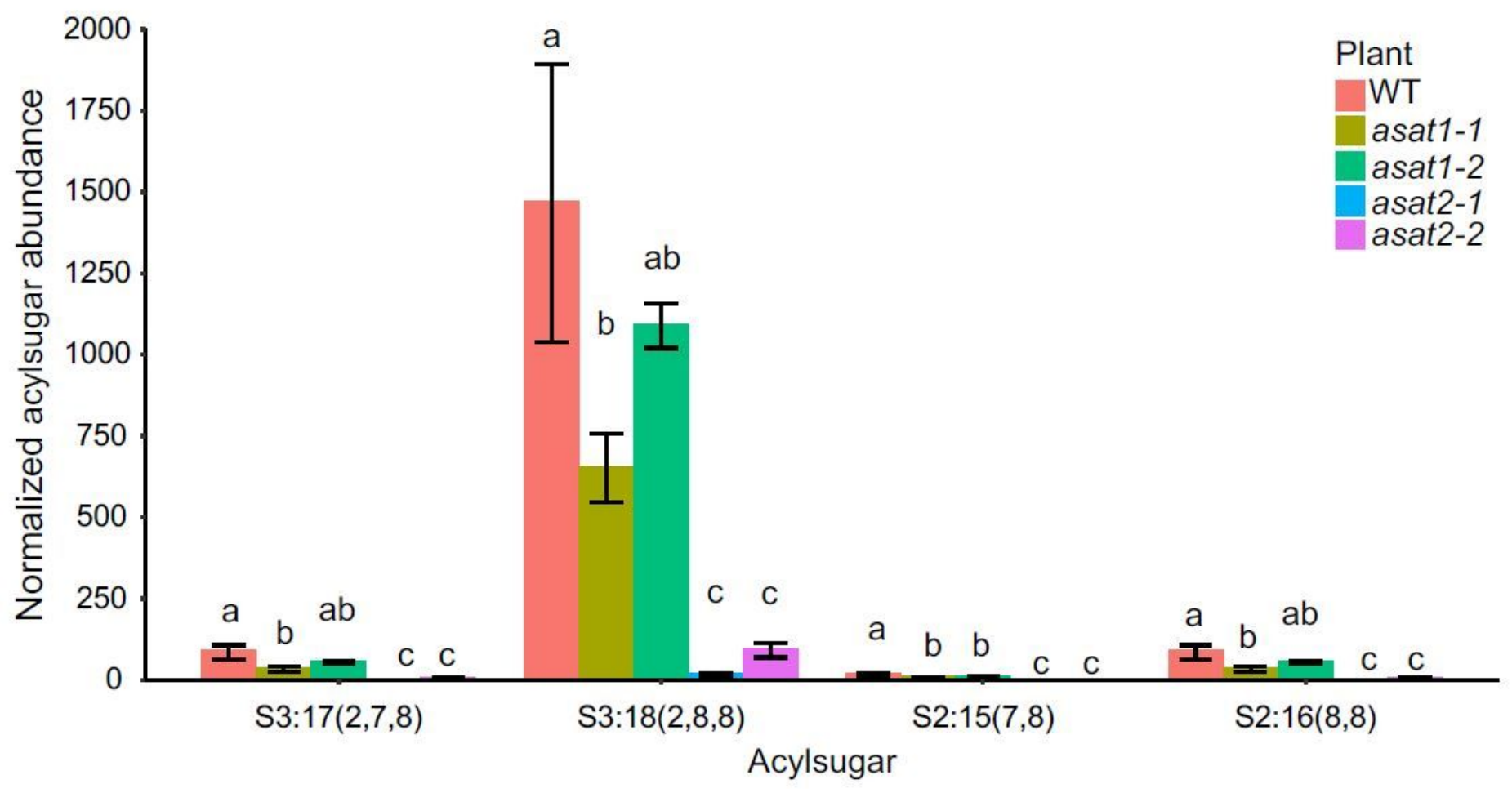

Figure 4

Abundance of two Nicotiana benthamiana acylsugars (S2:17(2,7,8) and S2:18(2,8,8)), and two likely pathway intermediates/fragmentations (S2:15(7,8) and S2:16(8,8)) Acyl sugar LC/MS peak areas were normalized relative to the peak area of Telmisartan, which was added as an internal control, and then to the leaf dry weight (per gram). Error bars represent standard errors from measurements of three plants of each genotype. Significant differences for each acylsugar between different genotypes were tested using one-way ANOVA followed by a Duncan's post hoc test $(p<0.05)$. Differences between groups are denoted with letters. 

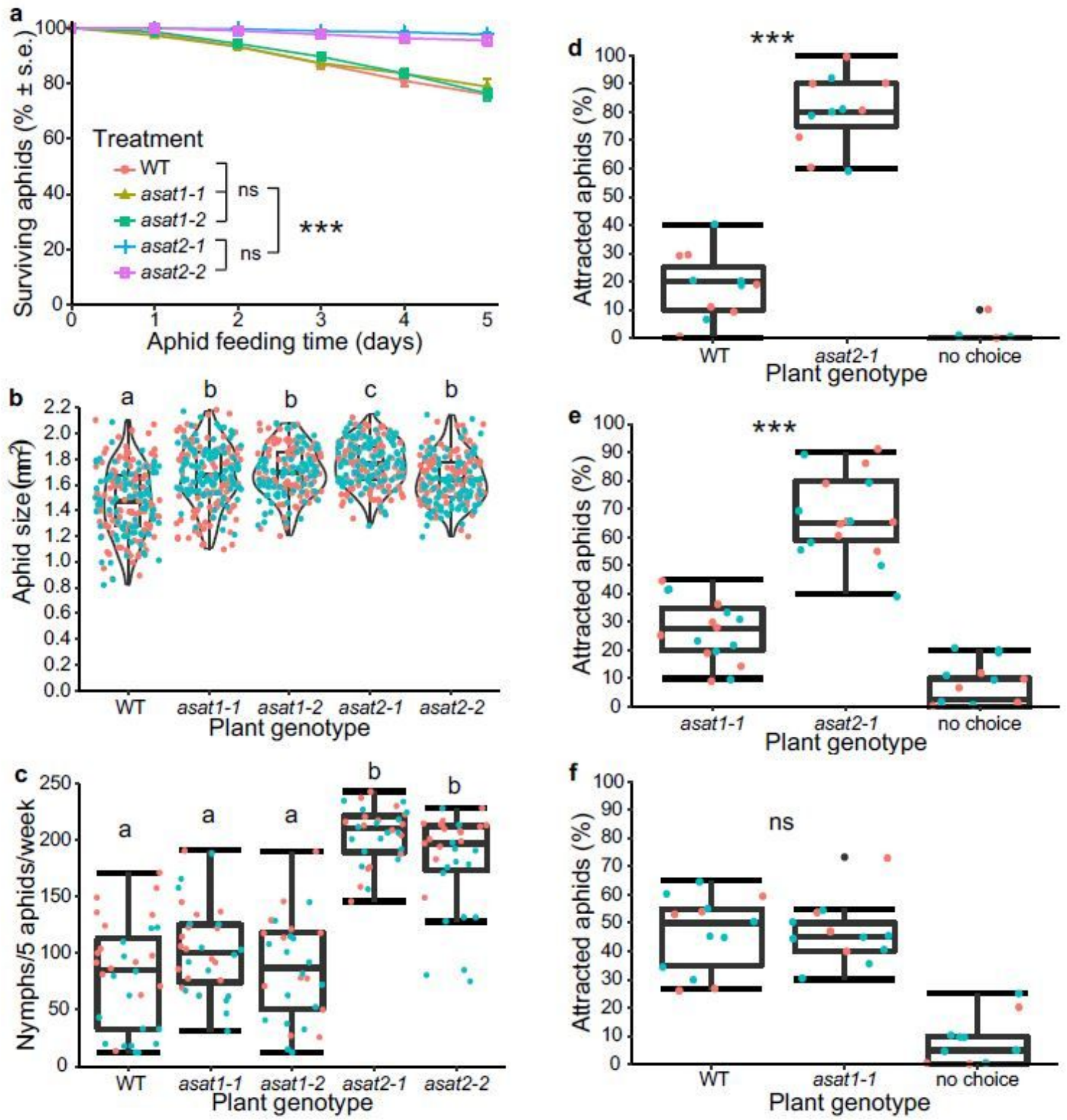

Figure 5

Myzus persicae bioassays with wildtype (WT), asat1, and asat2 Nicotiana benthamiana Data shown in all panels were combined from two independent experiments, which are shown in color orange and cyan in panels B-F. (a) Survival over 5 days of nymphs placed onto mutant and wildtype N. benthamiana. Significant differences are indicated for the 5-day time point: ns: not significant, $\star \star \star ~ p<0.001$, mean $+/-$ s.e. of $n=15-16$. Significant differences were tested using one-way ANOVA with a fixed factor of genotypes and a block effect of experiment followed by a Bonferroni post hoc test for multiple comparisons. Full statistical data for all time points are in Table S4. (b) Aphid growth, as measured by 
body size after 5 days feeding on N. benthamiana. (c) Aphid reproduction, as measured by the number of nymphs that were produced by five aphids in one week. Significant differences between different groups $(p<0.05)$ were determined using ANOVA with a fixed factor of genotypes and a block effect of experiment followed by a Duncan's post hoc test and are indicated by lowercase letters above each group in panels B and C. (d-f) Aphid choice among detached leaves of each plant genotype, significant differences between genotypes were assessed using Chi-square tests, $* \star \star p<0.001$; ns: no significant difference; no choice: aphids were elsewhere in the Petri dish and not on a leaf. The box plots show the median, interquartile range, maximum and minimum after removal of outliers, and the individual data points.
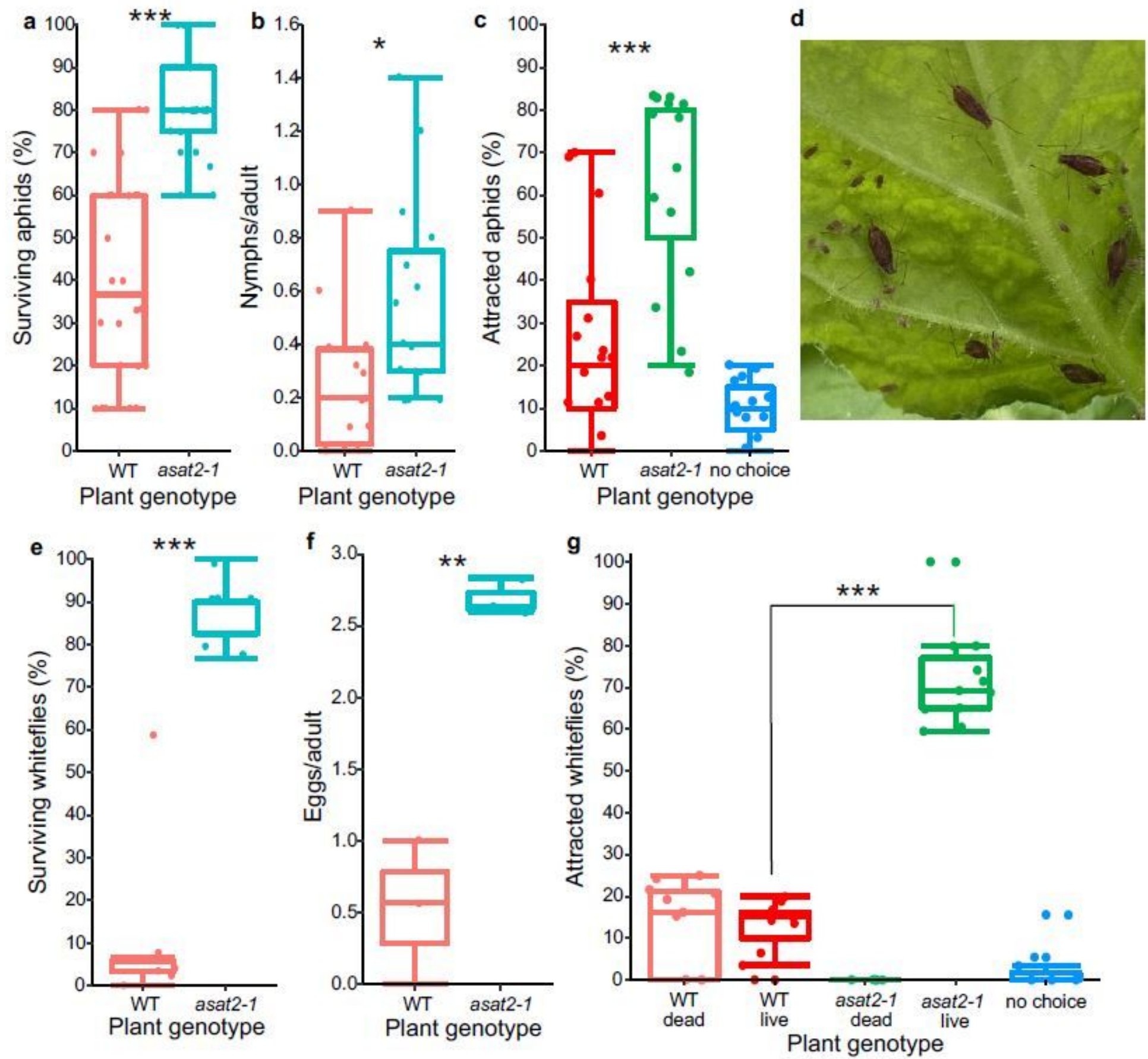


\section{Figure 6}

Potato aphid and whitefly bioassays on asat2-1 and wildtype (WT) N. benthamiana. (a-d) potato aphid (M. euphorbiae) bioassays. (a) aphid survival in 24 hours $(n=15)$. (b) aphid reproduction in 24 hours $(n=15)$. (c) aphid choices between detached leaves of each plant genotype $(n=15)$. (d) An established M. euphorbiae colony on an N. benthamiana asat2-1 leaf. (e-g) whitefly bioassays. (e) whitefly survival in 3 days $(n=6)$. ( $f$ ) whitefly reproduction measured as number of eggs produced per adults in 3 days $(n=3)$.

(g) whitefly choices between plants of each genotype ( $\mathrm{n}=3$ for 4 independent experiments). Significantly differences were tested using independent t-tests for aphid and whitefly survival and reproduction data. Chi-square tests were used for aphid and whitefly choice assays. ${ }^{*} p<0.05,{ }^{*} p<0.01,{ }^{* \star} p<0.001$; no choice: insects were not on a leaf at the end of the experiment. The box plots show the median, interquartile range, maximum and minimum after removal of outliers, and the individual data points.
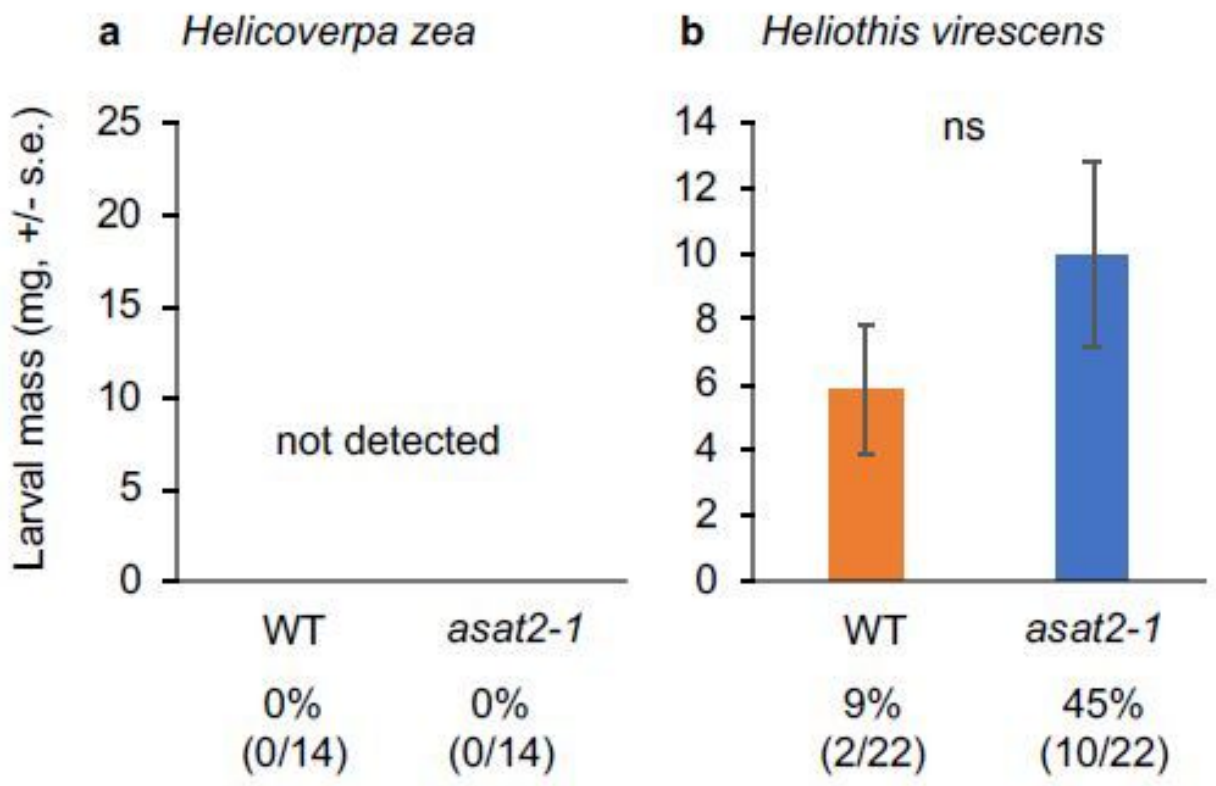

c Trichoplusia ni
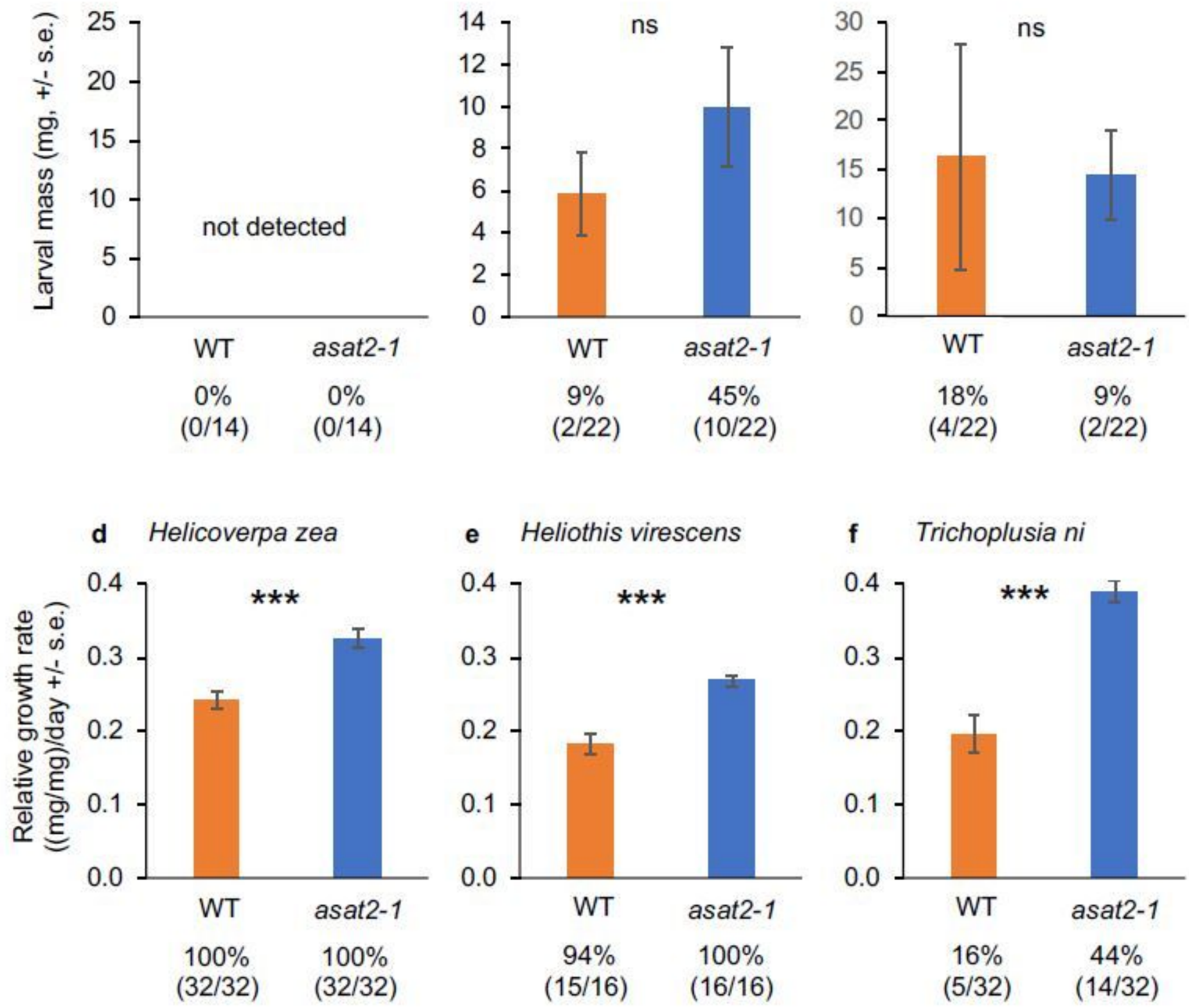

f Trichoplusia ni

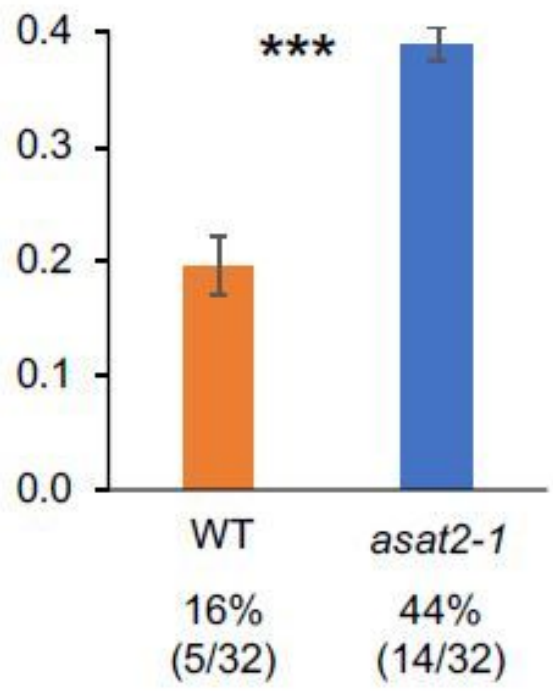


Figure 7

Caterpillar bioassays on wildtype (WT) and asat2-1 mutant Nicotiana benthamiana. (a, b, c) Larval mass of surviving Helicoverpa zea, Heliothis virescens, and Trichoplusia ni 10 days after being placed on plants as neonates. ( $d, e, f)$ Relative growth rate of surviving $H$. zea, $H$. virescens, and T. ni on wildtype and asat2-1 plants. Insects were raised for five days on artificial diet, prior to 7 days of feeding on $\mathrm{N}$. benthamiana. Percent survival (number of surviving insects/number of total insects) is shown below each figure. Mean $+/$ - s.e. , ${ }^{\star \star \star} p<0.001$, t-test; ns: no significant difference $(P>0.05)$.
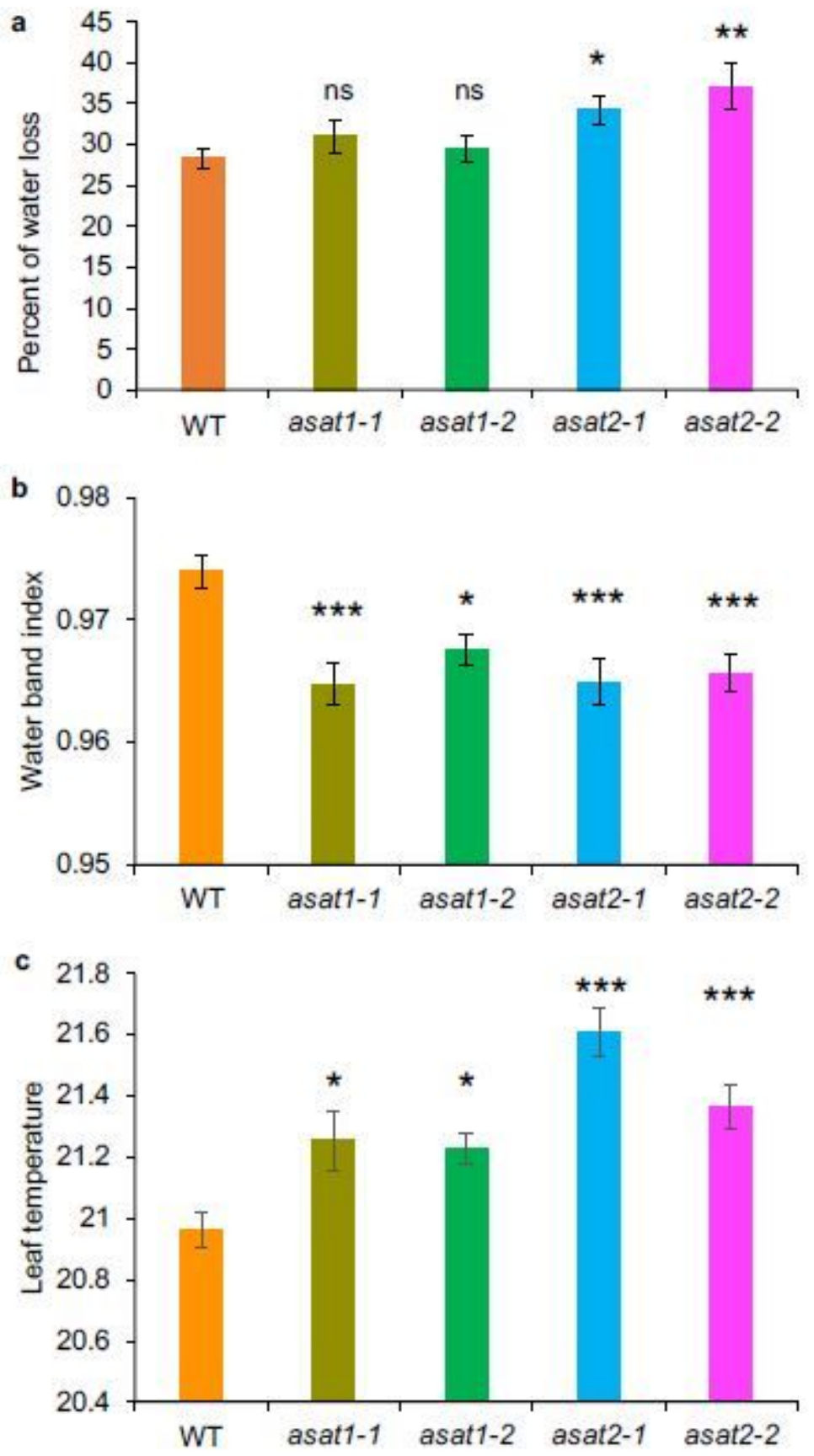

Figure 8 
Water loss and leaf temperature of wildtype (WT), asat1, and asat2 Nicotiana benthamiana. (a) Percent of water loss from detached leaves in 24 hours, mean $+/$ - s.e. of $n=15$. (b) Leaf water content measured by the water band index from hyperspectral imaging , mean $+/$ - s.e. of $n=20$. (c) Leaf temperatures from leaves of different plant genotypes, mean $+/$ - s.e. of $n=20$. $n s=$ no significant difference, ${ }^{*} p<0.05, * \star p<$ $0.01, * \star \star p<0.001$, Dunnett's test relative to wildtype control.

\section{Supplementary Files}

This is a list of supplementary files associated with this preprint. Click to download.

- SupplementaryFigures.pdf

- ASATTableS1SolASATs.xIsx

- ASATTableS2proteinsequences.docx

- ASATTableS3rawdata.xlsx

- ASATTableS4gRNAsPrimers.xIsx 